\title{
Knowledge and Practice Assessment In The Implementation of Malaria Primary Prevention in The Azire Health Area in Northwest Cameroon
}

\author{
Taah Oliver Fon ${ }^{1}$, Bodzewan Emmanuel Fonyuy ${ }^{2}$ Tata Fon Elvis ${ }^{3}$ \\ ${ }_{1}^{1}$ Training School for State Registered Nureses-Bamenda, Camernoon. \\ 2 Director, College for Registered Nurses-Bamenda, Lecturer Faculty of Health Sciences, \\ University of Bamenda, Cameroon. \\ ${ }^{3}$ Director, College of Senior Laboratory Technicians-Bamenda; Lecturer Catholic University \\ of Bamenda, Cameroon.
}

Email: ebodzewan@gmail.com

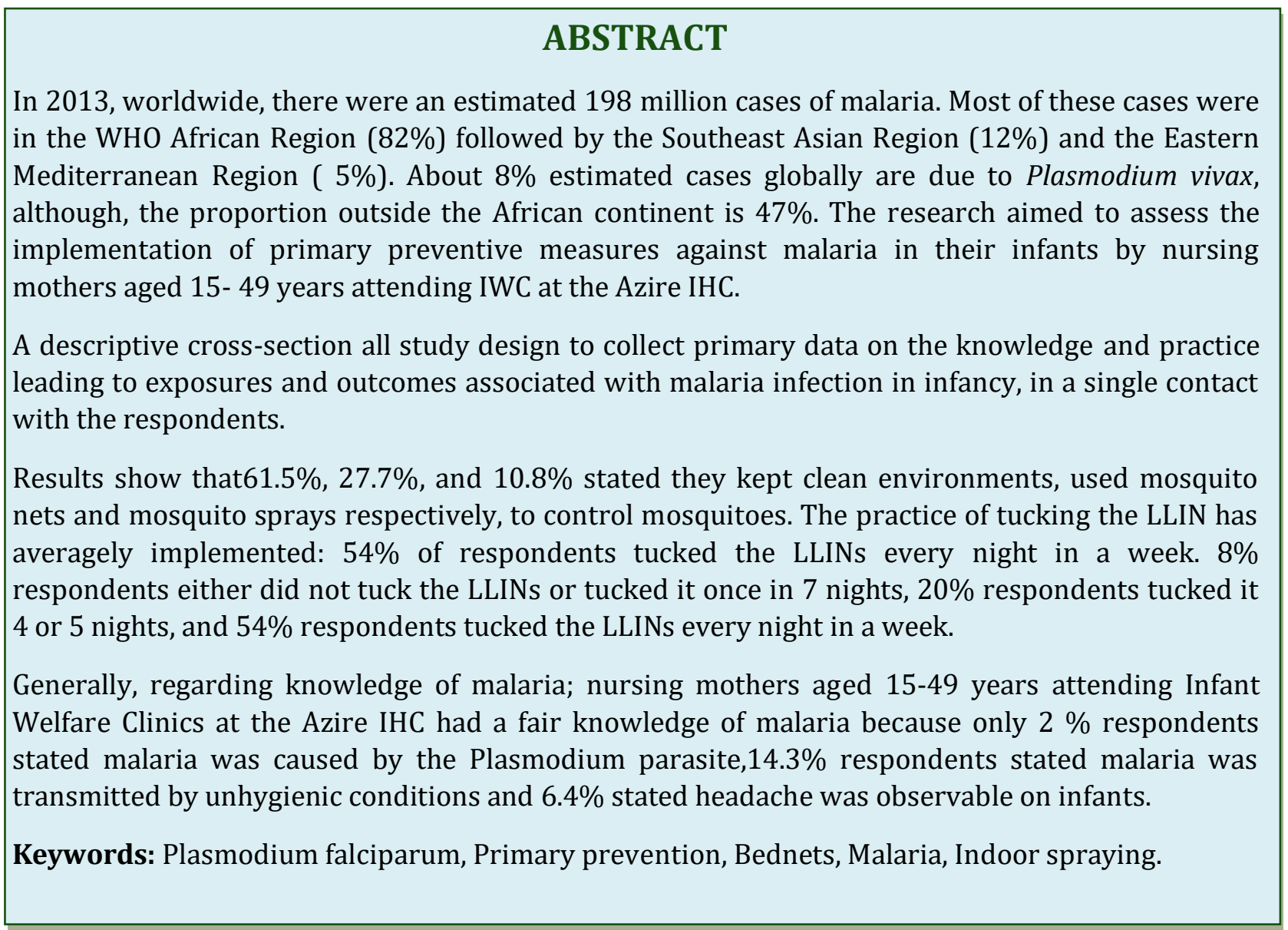

\section{Introduction}

The current epidemiologic report of malaria in terms of aetiology, transmissibility, current diagnostic and treatment options, global communication, and advocacy, the impact of therapeutic and preventive measures on incidence rates will be evaluated, before 
identifying gaps in certain areas requiring research and finally, in the light of the goals set by the WHO, a contextual discussion of this work will be done, in order to improve implementation of measures, so as to contribute to the achievement of the goals set by WHO before the stipulated deadlines.

In 2013, worldwide, there were an estimated 198million cases of malaria. Most of these cases were in the WHO African Region( 82\%) followed by the Southeast Asian Region $(12 \%)$ and the Eastern Mediterranean Region( 5\%). About 8\% estimated cases globally are due to Plasmodium vivax, although, the proportion outside the African continent is $47 \%$ (WHO, 2015).

It is estimated that 1.2 billion malaria cases and 6.2 million fewer malaria deaths occurred globally between 2001 and 2015 than would have been the case had incidence and mortality rates remained unchanged since 2000. In Sub-Saharan Africa, it is estimated that 943 million fewer malaria cases occurred and that malaria prevention interventions were responsible for $70 \%$ of this reduction, averting 663 million deaths (WHO,2016).

The proportion of children in Sub-Saharan Africa with Plasmodium falciparum malaria receiving ACT is estimated to have increased to $16 \%$ in 2014 . A major reason for this low percentage is that a high proportion of children with fever are not taken for the care, or use the informal private sector, where they are less likely to obtain ACT, for treatment within the WHO African Region (WHO, 2015).

The proportion of suspected malaria cases presenting for care in the public sector that received a malaria diagnostic test increased from $74 \%$ in 2005 to $78 \%$ in 2014 . The WHO African Region has had the largest increase in levels of malaria cases tested in 2005, to $41 \%$ in 2010 and to 65\% in 2014 (WHO, 2015).

The proportion of the population sleeping under an ITN increased from less than $2 \%$ in 2000 to an estimated 55\% in 2015. Ensuring access to ITNs has been critical to increasing the proportion of the population prevented from mosquito bites. Nearly 500 million ITNs were delivered to countries in Sub-Saharan Africa between 2013 and 2015 and the proportion of the population with access to an ITN increased to an estimated $67 \%$ in 2015(WHO, 2016).

Global financing for malaria control increased from an estimated USD 960 million in 2005 to USD2.5 billion in 2014. Of the total invested in 2014, international investments accounted for $78 \%$ and governments of malariaendemicc countries for 22\%. However, international funding for malaria control decreased by $8 \%$ and domestic funding increased by $1 \%$ between 2013 and 2014 (WHO, 2016).

The number of malaria deaths in children aged less than 5 years is estimated to have decreased from 723,000 globally in 2000 to 306,000 globally in 2015 and the proportion of the bulk of this decrease occurring in SubSaharan Africa. Reduction in malaria deaths has contributed substantially to progress towards achieving MDG Target 4A, which was to reduce the under- 5 mortality rate by twothirds between 1990 and 2015 (WHO, 2016).

Whereas only 13 countries were estimated to have fewer than 1000 malaria cases in 2000, 33 countries were estimated to have achieved this milestone in 2015. In 2014, 16 countries reported zero indigenous cases (WHO, 2016).

The vision of the WHO and the Global Malaria Community is a world free from malaria. The Global Technical Strategy for Malaria 2016- 
2030 sets the most ambitious yet feasible targets for reductions in malaria cases and deaths since the malaria eradication era. It also provides a framework for developing programmes that are tailored to local circumstances, with the aim of accelerating progress towards malaria elimination. Goals, milestones and targets of the Global Technical Strategy for Malaria 2016-2030 and Action and investment to defeat malaria 2016-2030:

1. Reduce malaria mortality rates globally compared with 2015at at least $40 \%$ in 2020 , at least $75 \%$ in 2025 and at least $90 \%$ in 2030.

2. Reduce malaria case incidence globally compared with 2015 by at least $40 \%$ in 2020 , at least $75 \%$ in 2025 and at least $90 \%$ in 2030.

3. Eliminate malaria from countries in which malaria was transmitted in 2015 globally compared with 2015 by at least $40 \%$ in 2020 , at least $75 \%$ in 2025 and at least $90 \%$ in 2030.

4. Prevent the re-establishment of malaria in all countries that are malaria-free (WHO, 2016).

According to the UNICEF Global Malaria Database, based on the 24 multiple indicator cluster surveys for Sub-Saharan Africa for 2000-2006, the percentage of children under five years with fever due to malaria receiving anti-malaria medicines, by location was as follows: $42 \%$ at home, $17 \%$ at home and health facility, and $42 \%$ at health facility.

Malaria transmission occurs in all six WHO regions. Globally, an estimated 3.3 billion people in 97 countries and territories are at risk of being infected with malaria and developing the disease, and 1.2 billion are at high risk ( $>1$ in 1000 chances of getting malaria in a year). According to the latest estimates, nearly half of the world population is at risk of malaria. In 2015, there were roughly 212 million malaria cases and an estimated 429000 malaria deaths. Increased prevention and control measures have led to a $29 \%$ reduction in malaria mortality rates globally since 2010. Sub-Saharan African continues to carry a disproportionately high share of the global malaria burden. In 2015, the region was home to $90 \%$ malaria cases and $92 \%$ malaria deaths including children aged less than five years who account for $78 \%$ of all deaths (WHO, 2018).

According to UNICEF Report (2007),between 350 million and 500million episodes of clinical malaria occur each year, leading to an estimated 1 million deaths, most in SubSaharan Africa and among children under age five accounting for 1 death in 10 (8\%) worldwide and nearly 1 death in $5(18 \%)$ in Sub-Saharan Africa; $15 \%$ of children under age five sleep under any type of mosquito net, the proportion of children under age five sleeping under an insecticide-treated net is $8 \% ; 18 \%$ of children under age five received anti-malarial medicines within 24 hours and $7 \%$ of Cameroon children under age five were sleeping under an insecticide-treated net .

A cross-sectional survey carried out in Guinea, the Gambia and Benin by Serignet al (2015) revealed : the overall prevalence of malaria in infants $\leq 6$ months was $11.8 \%$; the lack of attention to case management in this age group is a cause for concern and there is the need to enhance targeted preventive measures, adequate drug formulations, and treatment guidelines.

Malaria is endemic in Cameroon with a prevalence rate of $29 \%$. It is the cause of morbidity and mortality among the most vulnerable groups: children under five (18\%), pregnant women (5\%) people living with HIV/AIDS (5.5\%), and the poor (40\%). More than one million clinical cases of malaria occur annually, accounting for more than 
$50 \%$ morbidity among children under age five, 40 to $45 \%$ of medical consultations, and 30 to $47 \%$ of hospitalizations. In addition, it is responsible for $49 \%$ of prenatal consultations and $59 \%$ of hospitalizations during pregnancy leading to abortions, premature labour and deliveries and low birth weight (LBW), thereby resulting in infant and maternal mortality (Ndonget al, 2014).

Recent demographic and health surveys conducted in Cameroon have shown that the North West Region was among the most committed Regions of Cameroon in the fight against malaria (DHS, 2012).

Despite the progress in reducing malaria cases and deaths, malaria remains a major public health problem in many countries of the world. In Sub-Saharan Africa in 2014, some269 million of the 843 million people at risk of malaria lived in households without a single ITN or IRS, 15 million of the 28 million pregnant women at risk did not receive a single dose of IPTp, and between 68 and 80 million of the 92 million children with malaria did not receive ACT. As of 2014, the percentage of children receiving ACT in SubSaharan Africa was estimated at an increase of $16 \%$. A major reason for this low percentage is that a high proportion of children with fever are not taken for the care, or use the informal private sector, where they are less likely to receive ACTs for treatment. The ratio of staff to population is lowest in the countries with the highest numbers of malaria cases. The ability to finance an expansion of health services and to fill coverage gaps is constrained by lower national incomes in countries with the highest malaria burden. Plasmodium vivax is estimated to have been responsible for 13.8 million deaths of malaria cases globally in 2015 and to have accounted for about half the total number of malaria cases outside Africa. of the 78 countries reporting any monitoring data since2000, 60 reported resistance to at least one insecticide in one malaria vector population and 49 reported resistance to insecticides from two or more insecticide classes. The effectiveness of insecticide-based vector control is threatened as malaria mosquitoes develop resistance to the insecticides used in ITNs and IRS.LLINs, however, remain effective(though they may not be available, maybe poorly used or not used at all). Plasmodium falciparum resistance to artemisinin has now been detected in five countries. Malaria prevalence among children aged2-10 years is estimated to have decreased from $33 \%$ in 2000 to $16 \%$ in 2015(WHO, 2016).

\subsection{Research Question}

What are the primary preventive measures nursing mothers aged 15- 49 years attending IWC at the Azire Integrated Health Centre, implement to prevent malaria in their infants?

\subsection{Study Objectives}

\subsection{1.}

\section{General Objective}

To assess the implementation of primary preventive measures against malaria in their infants by nursing mothers aged 15- 49 years attending IWC at the Azire IHC.

\subsubsection{Specific Objectives}

- To assess the knowledge of nursing mothers aged 15- 49 years attending IWC at the Azire IHCon the basic notions of primary prevention of malaria on their infants.

- To find out the measures aimed at preventing malaria in their infants 
used by nursing mothers aged 15- 49 years attending IWC at the Azire IHC.

- To find out the difficulties faced by nursing mothers aged 15- 49 years attending IWC at the Azire IHC in the implementation of more effective primary preventive measures against malaria on their infants.

\subsection{Hypothesis}

Nursing mothers aged 15-49 years attending IWC at the Azire IHC who are knowledgeable of the effects of malaria on their infants would implement more effective primary preventive measures against malaria on their infants than those who are not

\subsection{Significance of the Study}

This study is intended to improve the knowledge and behavior of nursing mothers aged 15-49 years attending IWC at the Azire IHCon the implementation of primary preventive measures against malaria in their infants. It will guide them to modify their behavior using simple, affordable and cheap methods to prevent malaria in their infants more effectively. It will also provide reliable and valid baseline findings that can incite advocacy and competent organizations to sponsor large-scale research with more representative attributes in order to profitably modify public health policy and interventions within the framework of eliminating malaria from the North West Region and from the country as a whole.

\subsection{Scope of the Study}

This research tagged"An assessment of the knowledge and practice in the implementation of primary preventive measures against malaria on their infants by nursing mothers aged 15-49 years attending IWC at the Azire IHC" was carried out at the
Azire IHC from November 2017 to May 2018 by the investigator ,a third-year student of TSSRN Bamenda with focus on three areas of implementation of primary preventive measures against malaria on infants ,namely : preventing mosquito bites by the use of barrier methods especially LLINs; chemoprophylaxis in pregnancy; and maintaining basic sanitation.

\subsection{Limitations of the Study}

There was no standardization of respondents with respect to the criteria evaluated in the research and there were also no controls for each variable assessed. Findings were based on clients' judgments and reports. The sociopolitically unfriendly environment hindered evaluation of the liaison between the reports and the actual practice by each respondent. It was impossible to obtain a sample that was equally representative by age, education, job, ethnic group, geographic location of residence, genetic constitution and purchasing power. Also, the sample size would have been doubled in order to ensure more representative results if there were sufficient means and time.

\section{Methodology}

\subsection{Description of the Study Area}

The research was carried out at the Azire Integrated Health Centre, found in the Azire Health Area (AHA)from November 2017 to May 2018. The AHA is one of the nineteen health areas in the Bamenda Health District. The AHA is bounded to the north by the Ntamulung Health Area, to the South by the Atuakum Health Area, to the East by the Ntombab Health Area and to the West by the Alakum Health Area. It is found in the Bamenda Two Subdivision in the North West 
Region of Cameroon and the Health Centre is located inNitop 4 Quarter. It has a population of 72000 inhabitants and it is made up of 10 quarters or zones, namely; AzireA, Azire B, Lower AtouAzire, Nitop One, NitopTwo,Nitop Three, Nitop Four (where the leading health post is),Ntarikon One, NtarikonTwo, and Ntaturu. Each zone has a zonal representative or mobilizer who coordinates the various health activities in his or her zone.

\subsection{Study Design}

The investigator employed a descriptive cross-section all study design to collect data from 50 eligible and voluntary respondents in March and April 2018, to achieve the objectives of the research. The investigator used the design to collect primary data on the knowledge and practice leading to exposures and outcomes associated with malaria infection in infancy, in a single contact with the respondents.

\subsection{Study Population and Target Population}

The target population is infants. The independent variable is infant nursing mothers aged 15-49 years, for whom interventions to communicate behavior modification to enhance implementation of primary preventive measures against malaria on infants were directed. The dependent variable is infants. The study population is nursing mothers aged 15-49 years nursing an infant by the time of the research in particular, expectant and procreative women in general.

\subsection{Sample Size}

Nursing mothers aged 15-49 years of the Azire Integrated Health Area is the sample from which a sample size of 50 eligible respondents decided to participate, by signing an informed consent form after the investigator made a brief explanation of the purpose of the research. A sample size of fifty nursing mothers not living more than $2500 \mathrm{~m}$ from the Azire IHC completed the questionnaires from which data were analyzed

\subsection{Sampling Method}

The investigator used the simple random sampling method in which he did secret balloting of available respondents who had given their consent, based on the age ranges of the respondents' babies.

The investigator used a structured questionnaire to collect relevant data from 50 respondents.

\subsection{Data Collection Procedure}

The investigator directly administered the questionnaires himself to 50 respondents who willingly consented to participation after considering the explanation the investigator gave on the purpose of the research.

\subsection{Data Analyses Method}

Primary data was collected from 50 respondents using the structured questionnaire. They were then tallied, analyzed and presented the data on tables and figures using Microsoft word. 


\section{Results}

\subsection{Socio-Demographic Data}

4.1.1 Age of respondents:

\section{Frequency}

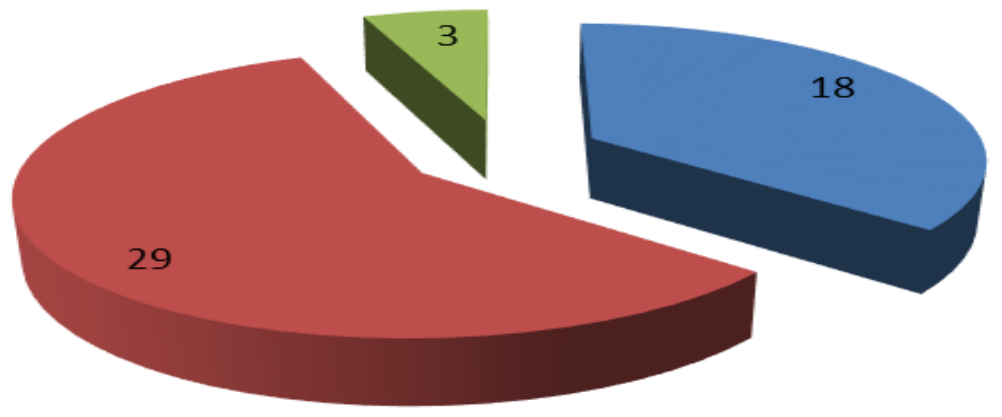

15-25 years

- 25-35years

- 35-49 years

Figure 2: Distribution of respondents according to age range:

From figure $2,36 \%$ of the respondents were between $15-25 y$ rs, $58 \%$ of them were between 25 35years and $8 \%$ felt between 35-49 years range.

\subsubsection{Age of respondents' infants}

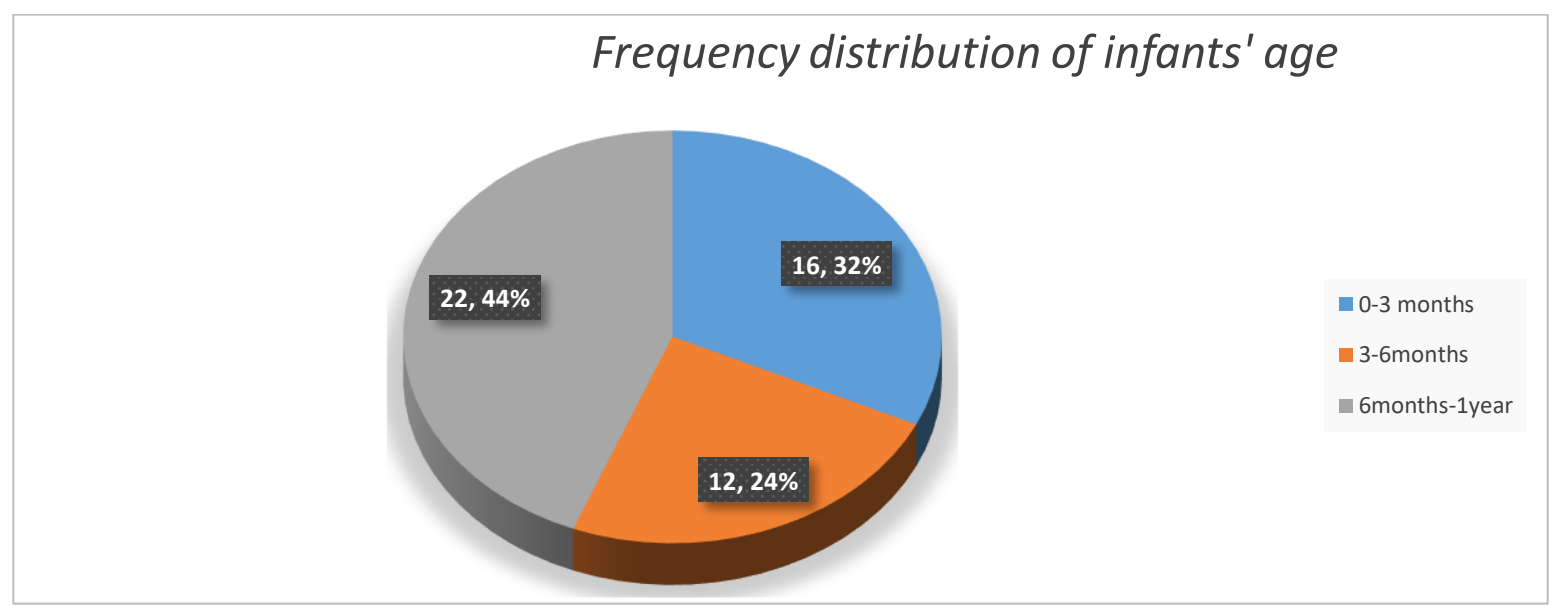

Figure 3: Distribution of respondents according to infant's age range:

Thirty-two percent of the respondents were within the age range $0-3$ months, $24 \%$ of them between 3-6months, and 44\% were within 6 months-1year age range. 


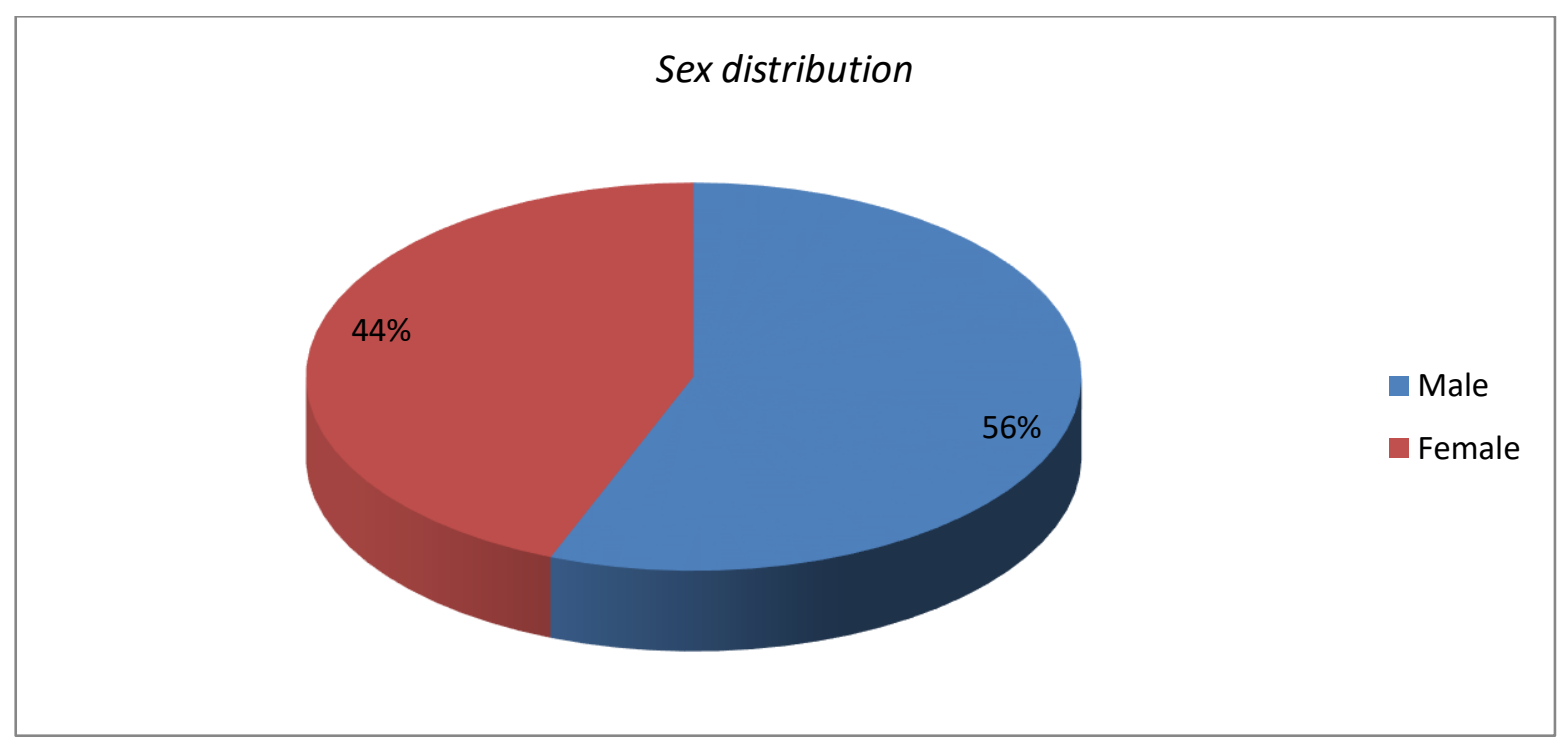

4.1.2 Sex of the respondents' infants:

Figure 4: Distribution of respondents according to sex of the infant: Fifty-six percent of infants were males $56 \%$ with $44 \%$ females.

4.1.2 Level of education of respondents:

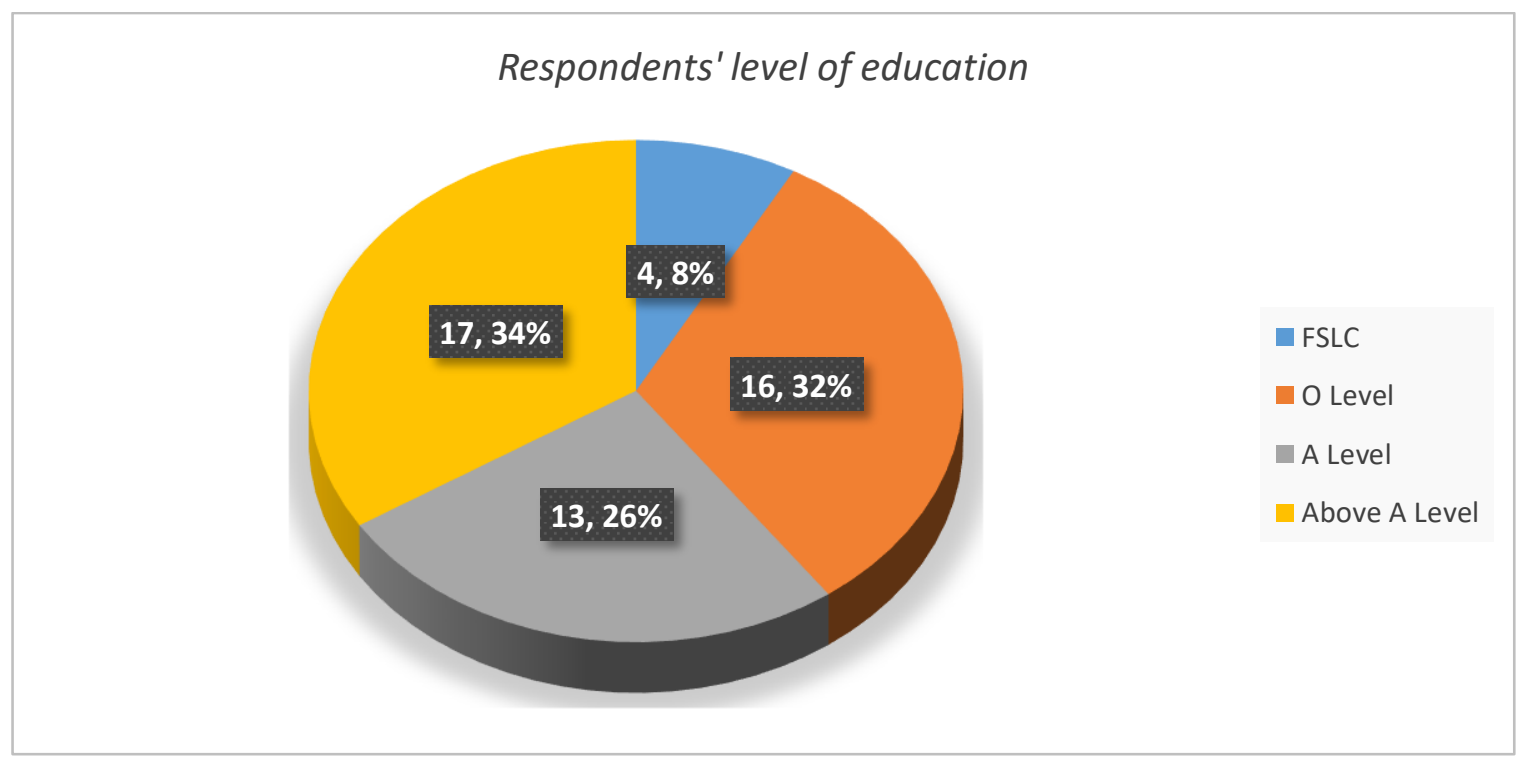

Figure 5: Distribution of respondents according to level of education:

Eight percent of the respondents had obtained the FSLC ; 32\% had obtained Ordinary Level certificate , $26 \%$ had obtained Advanced Level and 34\% had obtained a post-secondary school certificate. 
4.1.2 Respondents' ethnic groups

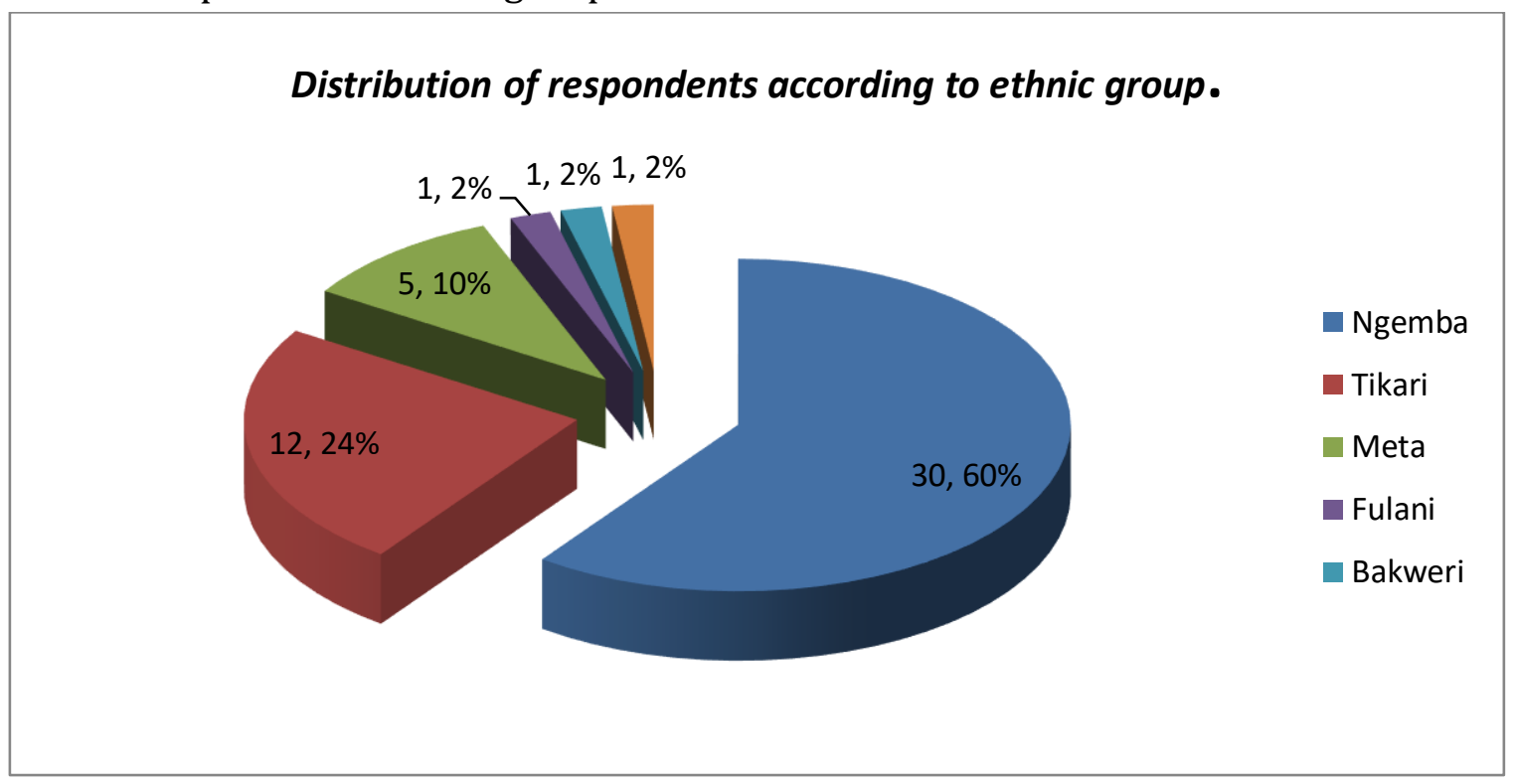

Figure 7: Distribution of respondents according to ethnic group:

Sixty percent of respondents were Ngemba, $24 \%$ were Tikari, $10 \%$ ) were Meta, $2 \%$ were Fulani, Bakweri and Bamileke respectively.

4.1.7 Religious affiliation of respondents:

\section{Frequency}

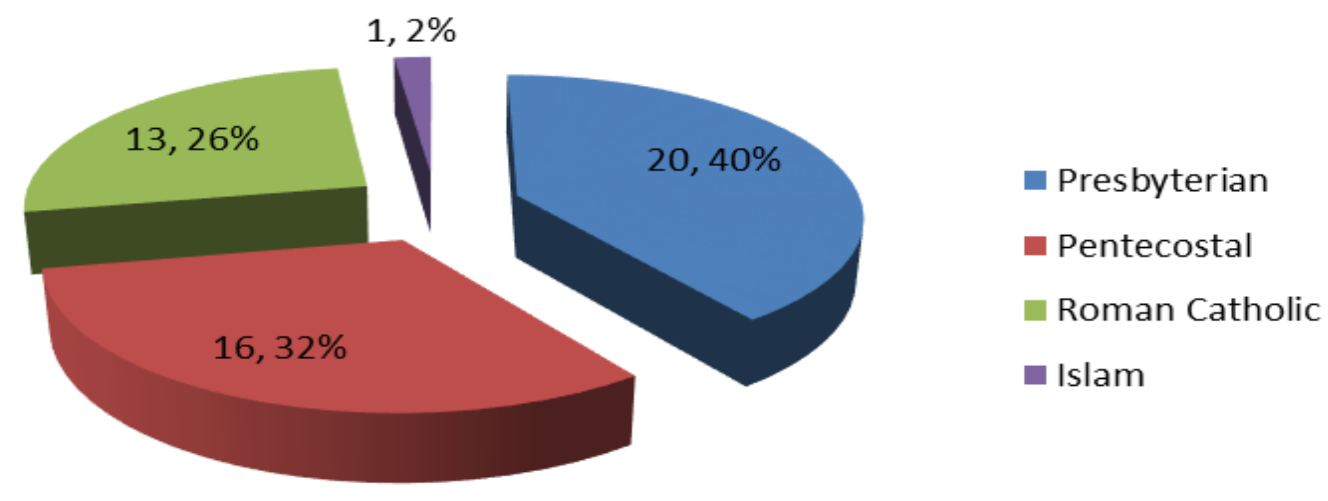

Figure 8: Distribution of respondents according to religious affiliation:

Forty percent of them were Presbyterian, 32\% were Pentecostal, 26\% were Roman Catholic and 2\% were Muslim. 


\subsubsection{Number of children per respondent}

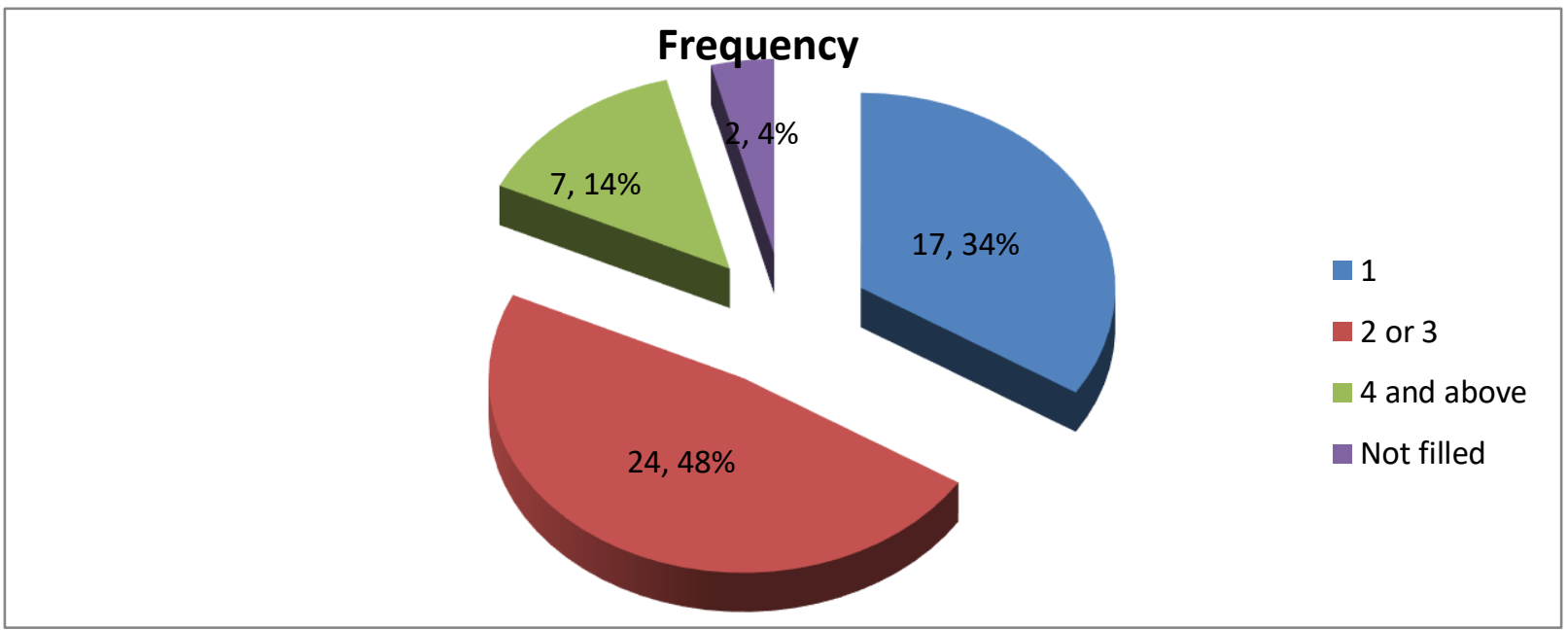

Figure 11: Distribution of respondents according to each respondent's parity: Thirty-four percent of respondents had one child, $48 \%$ had three children, $14 \%$ had 4 or more children. 4.1.10 Person(s) responsible for settling infant's health bills:

\section{Frequency}

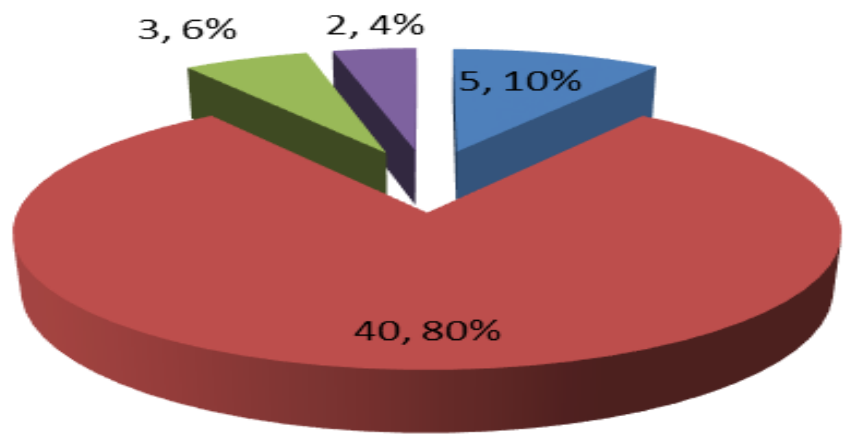

Mother

- Father

Both parents

Not filled

Figure 12: Distribution of respondents according to person(s) responsible for settling infant's health bills;

5 respondents (10\%) stated infant nursing mothers were responsible, 3 respondents $(6 \%)$ stated both parents were responsible, 40 respondents (80\%) stated that the father of the infant was responsible, 2 respondents (4\%) did not state who was responsible. 


\subsubsection{Place of infant birth}

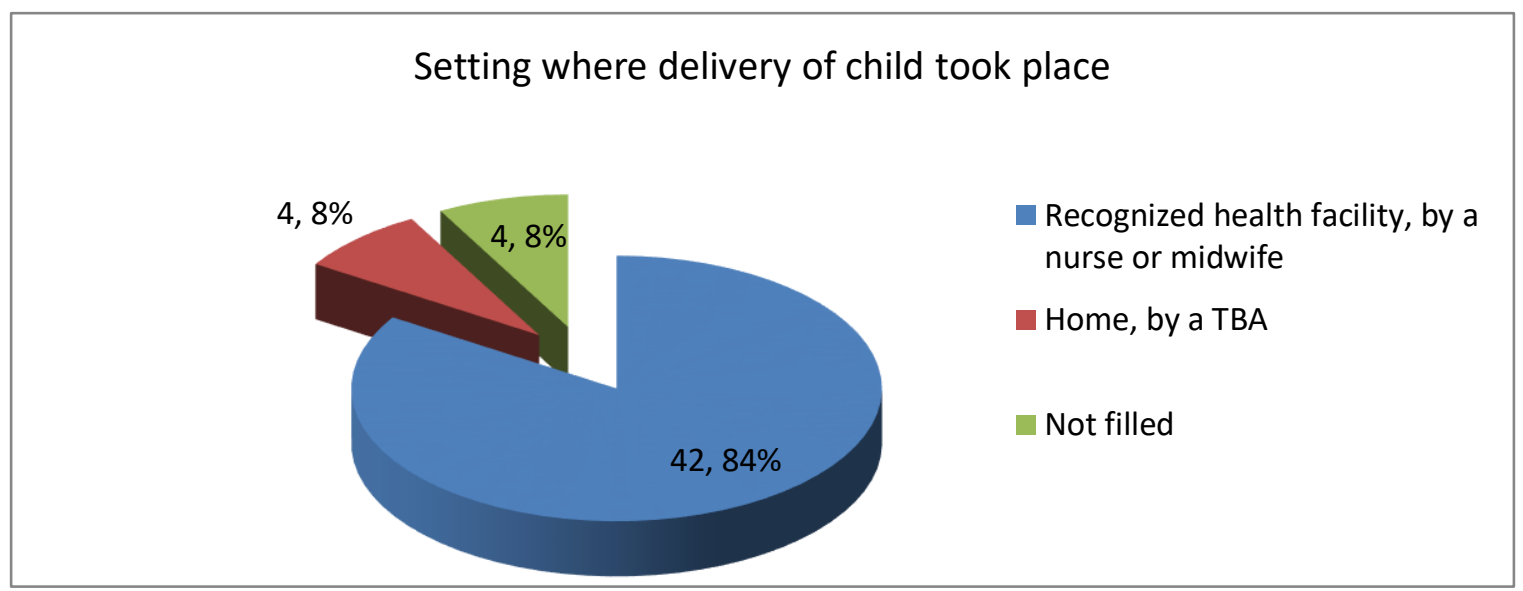

Figure 13: Distribution of respondents according to place of infant birth:

42 respondents (84\%) were delivered of their infants at a recognized health facility, 4 respondents (8\%) were delivered of their infants at home and 4 respondents (8\%) did not fill data on the place of infant birth.

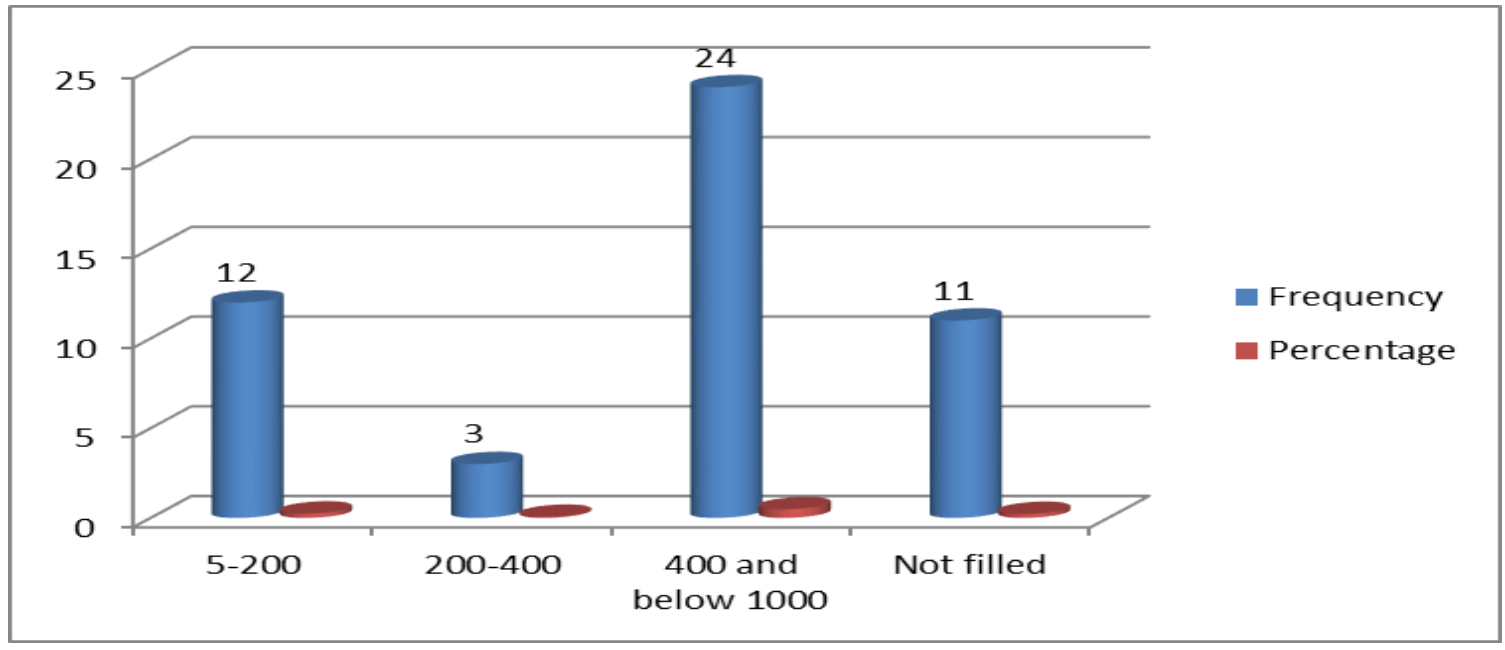

4.1.12 Approximate distance (in meters) between the home of each respondent and health facility of birth of the infant:

Figure 14: Distribution of respondents according to approximate distance between the home and health facility of birth of the infant; 12 respondents (24\%) said that the distance was between $5-200 \mathrm{~m}, 3$ respondents (6\%) said that the distance was between $200-400 \mathrm{~m}, 24$ respondents $(48 \%)$ said that the distance was between $400 \mathrm{~m}$ and below $1000 \mathrm{~m}$ and 11 respondents $(22 \%)$ did not fill data on the distance. Distance determines accessibility to health services. 


\subsection{General Notions Harboured About Malaria}

\subsubsection{Definition of malaria:}

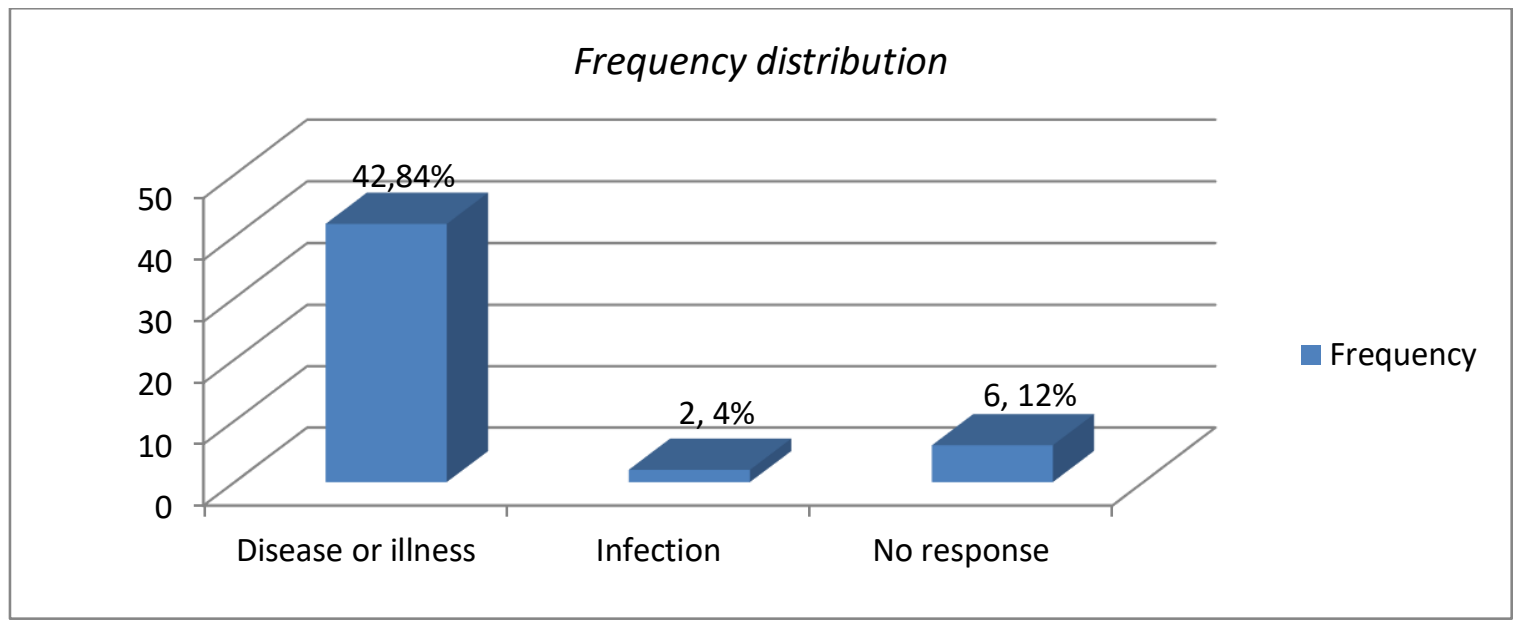

Figure 15: Distribution of respondents according to definition of malaria:

Eighty-four\% of the respondents defined malaria as a disease or illness, $4 \%$ defined malaria as an infection, and $12 \%$ had no knowledge on the definition of malaria.

\subsubsection{Causes of malaria}

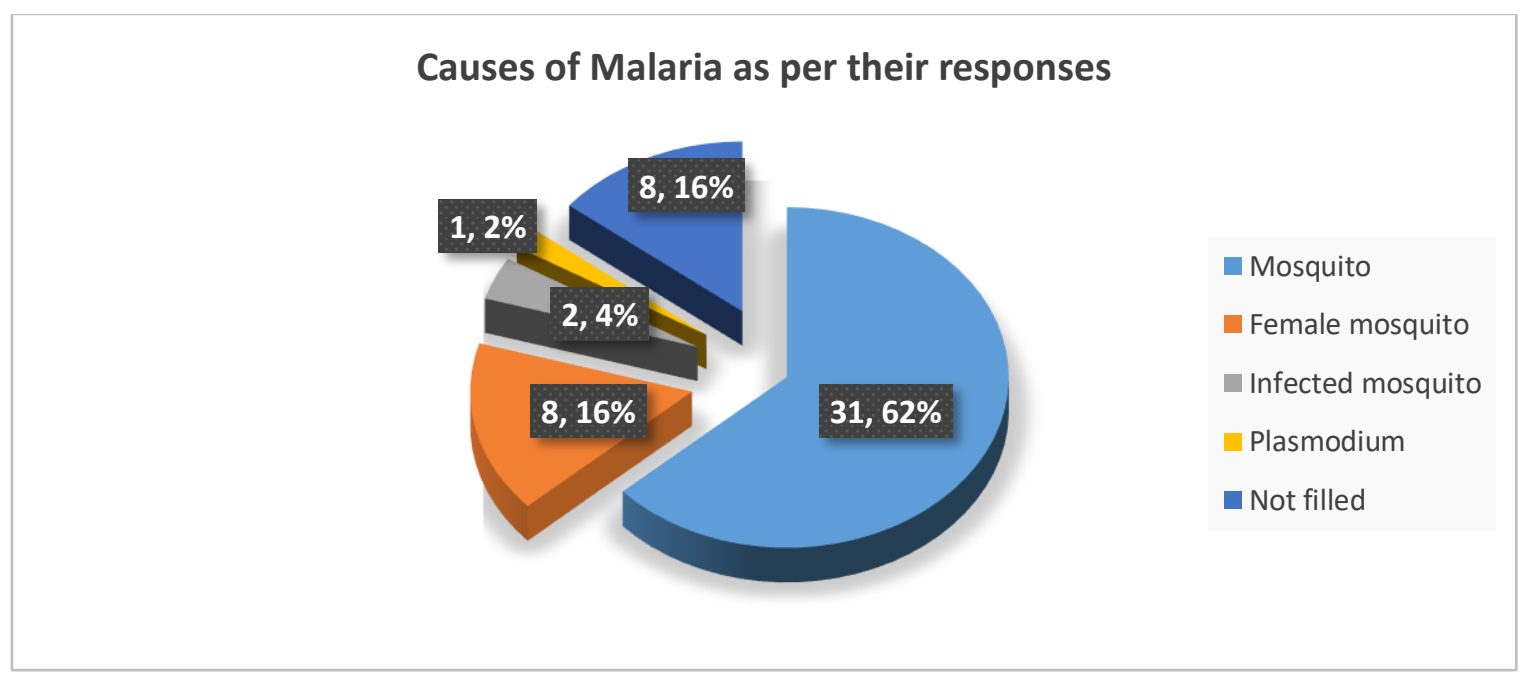

Figure 16: Distribution of respondents according to causes of malaria:

Sixty-two $\%$ of respondents stated that malaria was caused by mosquitoes, $16 \%$ stated malaria was caused by Female mosquitoes, 4\% stated malaria was caused by infected mosquitoes, 2\% stated malaria was caused by the Plasmodium parasite, $16 \%$ did not have an idea on the cause of malaria. 
4.2.4 Identification of control measures nursing mothers implement against mosquitoes.

\section{Control measures against mosquito bites}

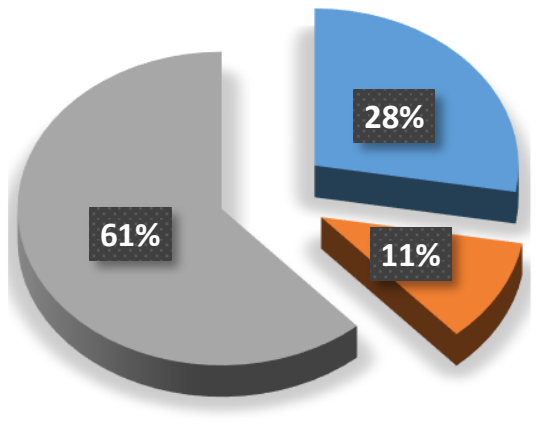

- Using a mosquito net

- Using a mosquito spray

Keeping clean environments

Figure 18: Distribution of respondents according to control measures they implement against mosquitoes, by frequency of responses:

$27.7 \%$ of respondents stated they used mosquito nets to control mosquitoes, $10.8 \%$ stated they used mosquito sprays and $61.5 \%$ stated they kept their environments clean to control mosquitoes.

\subsubsection{Probable signs of suspected malaria infection observable in infants}

\section{Probable signs to suspect malaria in infants}

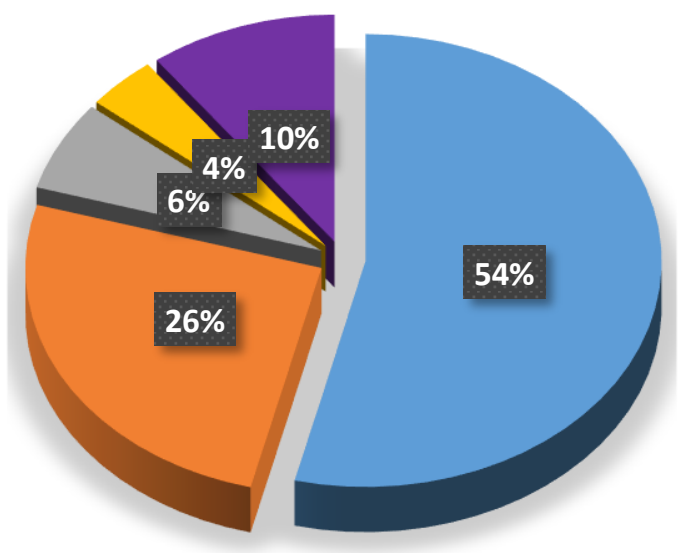

Fever

Vomiting

Headache

Crying

Inactivity

Figure 19: Distribution of respondents according to three likely signs of suspected malaria infection observable on infants.

$53.9 \%$ of respondents stated fever was most likely to manifest in infant malaria, $25.6 \%$ stated vomiting, $6.4 \%$ stated headache, $3.8 \%$ stated crying, and $10.3 \%$ stated inactivity were observable. 
4.2.6 History of previous infectious exposure of the infant to malaria:

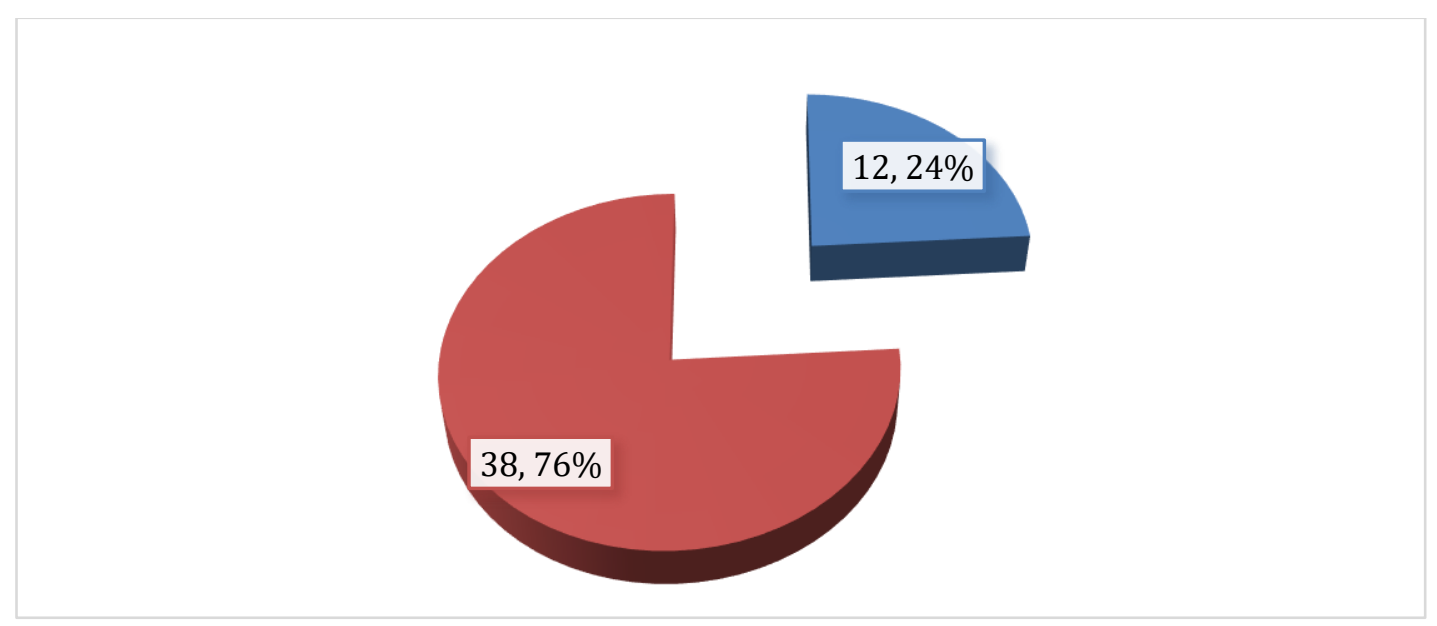

Figure20: Distribution of respondents according to knowledge of history of previous infectious exposure of the infant to malaria:

$24 \%$ of the respondents stated their infants had had a previous infectious exposure to malaria and $76 \%$ stated theirs had never had any malaria infection.

4.2.7 History of previous exposure of the infant to malaria by age range:

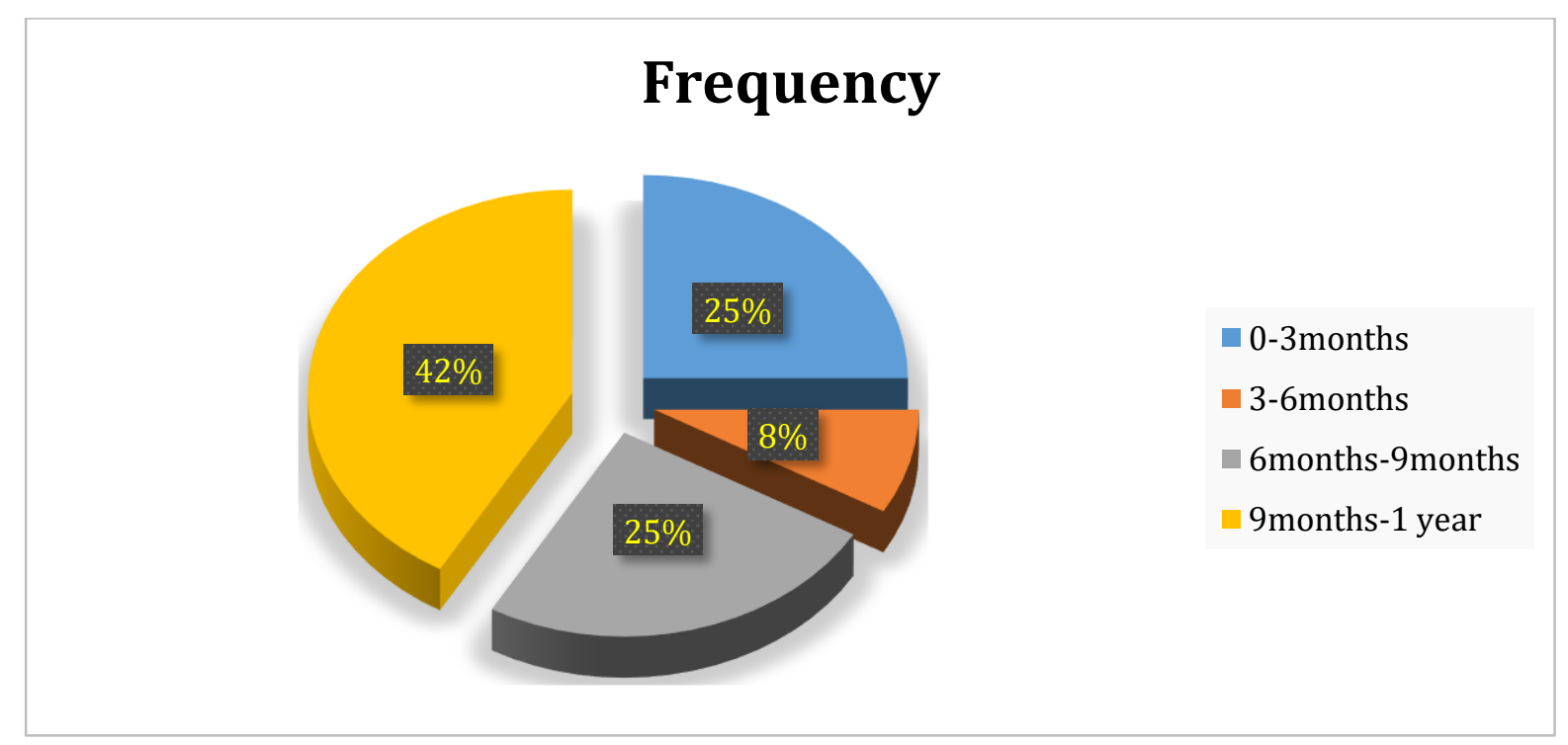

Figure 21: Distribution of respondents according to history of previous exposure of the infant to malaria by age range:

$25 \%$ of infants who were within the age range 0 -3months had had previous infectious exposures to malaria, $8.3 \%$ were within the age range $3-6$ months, $25 \%$ within the age range $6-9$ months, and $41.7 \%$ who were within the age range $9-12$ months, had experienced previous infectious exposures to malaria. 


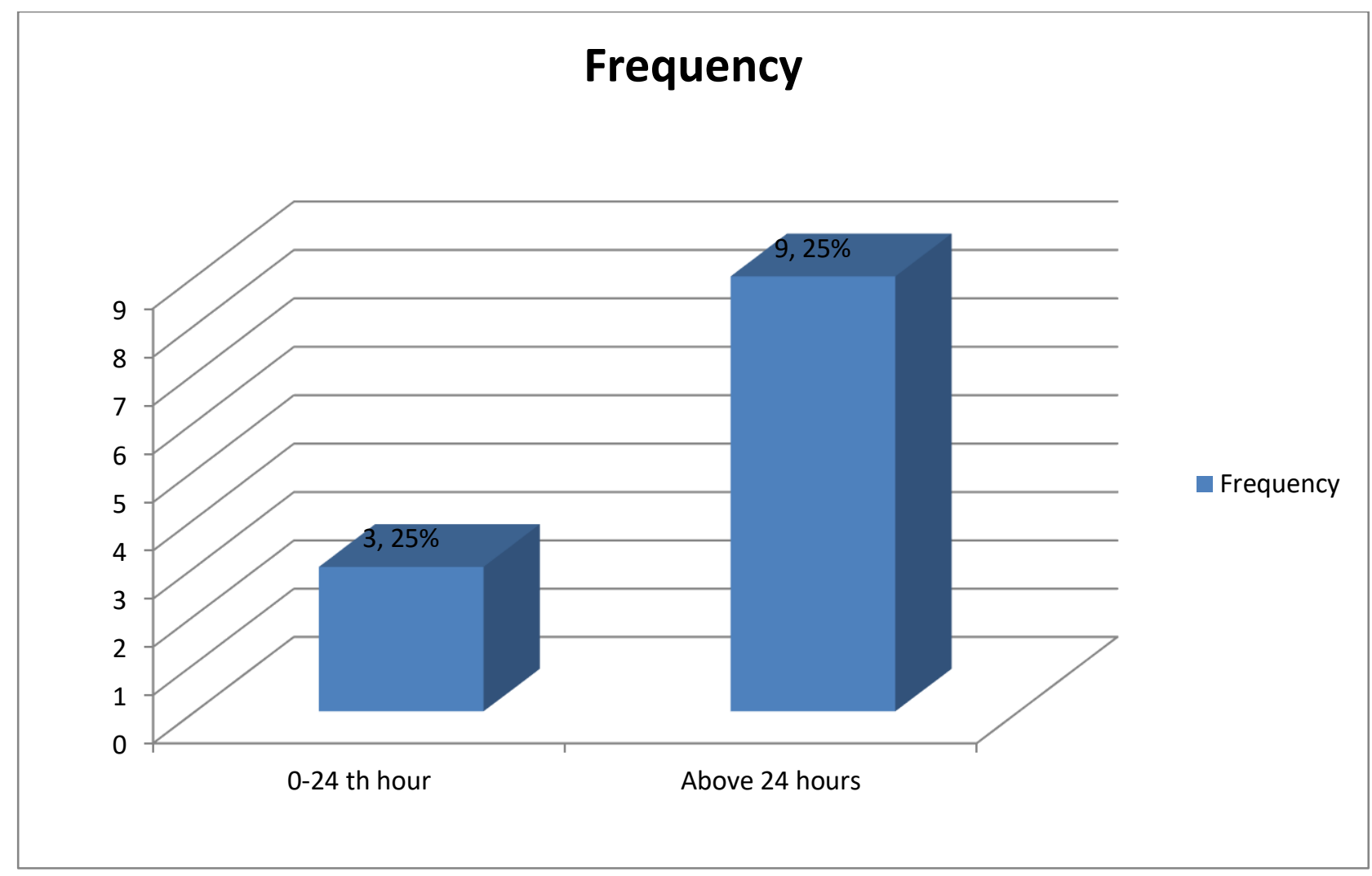

4.2.10 Duration from the time of observation of fever on the infant till when health care for the infant was sought:

Figure 25: Distribution of respondents with history of exposure, according to duration from observation of fever on infant till when health care was sought: $25 \%$ of them stated they sought health care for their infants within 24 hours of observation of fever $75 \%$ stated they sought health care for their infants after 24 hours of observation of fever on the infant.

4.2.13 Where the nursing mothers sought health care for the infants suspected of malaria infection:

\begin{tabular}{|l|c|c|}
\hline Place where care was sought & Frequency & Percentage \\
\hline Recognized Hospital & 12 & $100 \%$ \\
\hline Tradi-practitioner & 0 & 0 \\
\hline Total & 12 & 100 \\
\hline
\end{tabular}

The above table shows the distribution of respondents with history of exposure of infant, according to where they sought health care for the infant suspected of malaria infection: $100 \%$ stated they sought health care for the infant suspected with malaria infection at recognized health facilities and none sought care at the tradi-practitioners. 


\section{Frequency distribution realtive to season of transmission.}

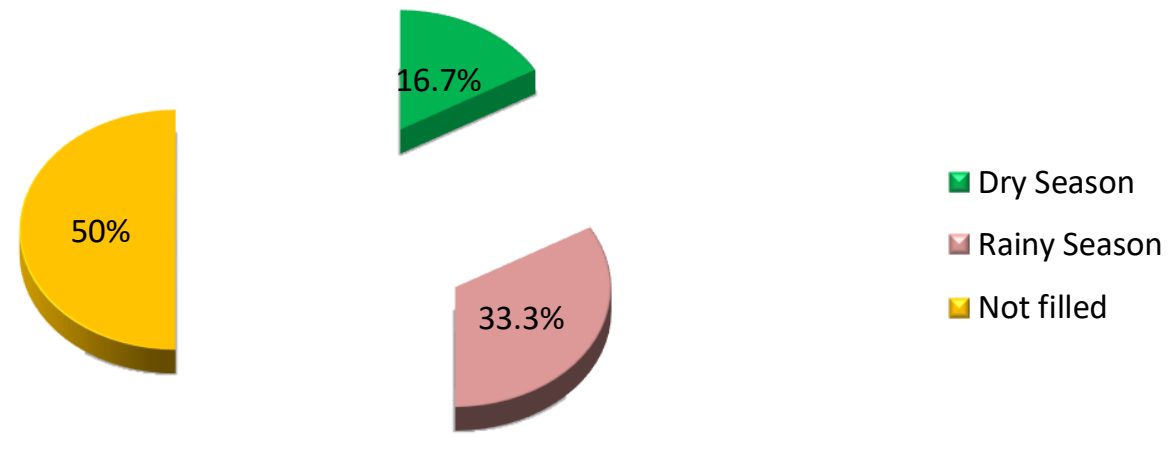

4.2.15 Season in which the infant malaria case occurred:

Figure 28: Distribution of respondents with history of exposure of the infant, according to the season in which the infant malaria case occurred: $16.7 \%$ and $33.3 \%$ respectively stated malaria infection of their infants occurred in the Dry Season and Rainy Season.

4.2.16 Approximate duration of the incubation period of Plasmodium after an infectious blood meal of a susceptible infant:

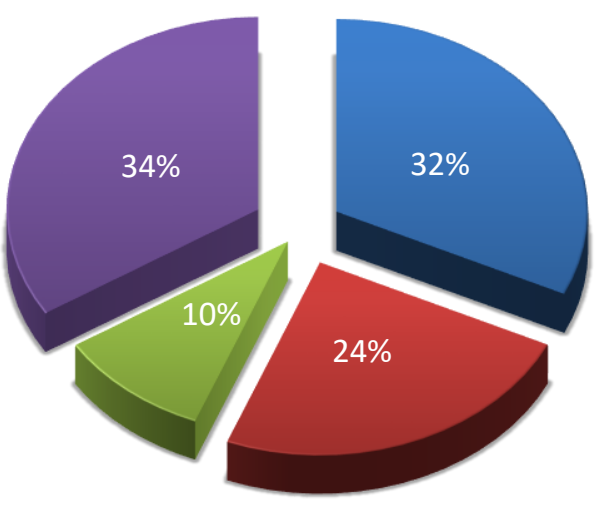

$\square$ 0-7 days

7-28days

$\square$ I do not know

$\square$ Not filled

Figure 29: Distribution of respondents, according to approximate duration of the incubation period of Plasmodium after an infectious blood meal of a susceptible infant: 
$32 \%$ of the respondents stated signs and symptoms following an infectious bite of an infant by an infected female Anopheles mosquito, could be observed within 7 days of the bite. $24 \%$ stated signs could be observed between the $7^{\text {th }}$ and the $28^{\text {th }}$ day after the bite; $10 \%$ did not know the incubation period.

4.2.18 Knowledge of anyone who lost her baby due to malaria:

\section{Frequency}

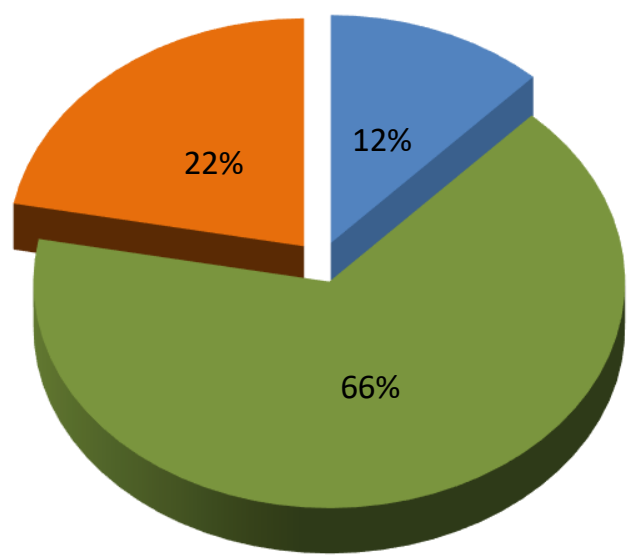

- Yes

No

No response

Figure 31: Distribution of respondents according to knowledge of anyone who lost her baby due to malaria:

$12 \%$ stated they knew someone who had lost her infant due to malaria while $66 \%$ stated they never knew anyone.

\subsection{Primary Preventive Interventions}

4.3.1. Medication to Prevent Malaria:

\section{Frequency of responses}

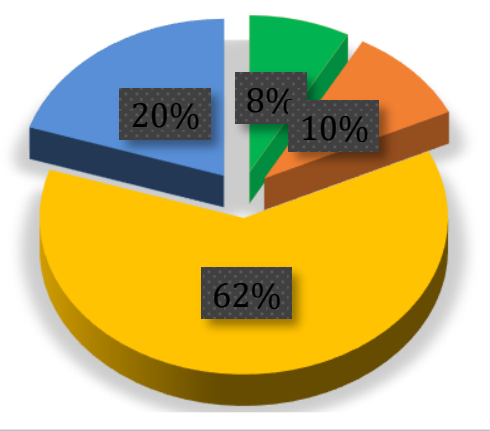

$$
\begin{aligned}
& \square 1 \\
& \square 2 \\
& =3 \text { or } 4 \\
& \square \text { Forgotten }
\end{aligned}
$$

Figure 33: Distribution of respondents according to frequency of fansidar administration during the same pregnancy: $8 \%$ of them stated Fansidar was administered once, 10\% stated Fansidar was 
administered twice $62 \%$ stated Fansidar was administered 3 or 4 times in the same pregnancy; $20 \%$ stated they had forgotten how many times Fansidar was administered.

4.3.1.4 Gestational age at the first administration of Fansidar:

\section{Frequency}

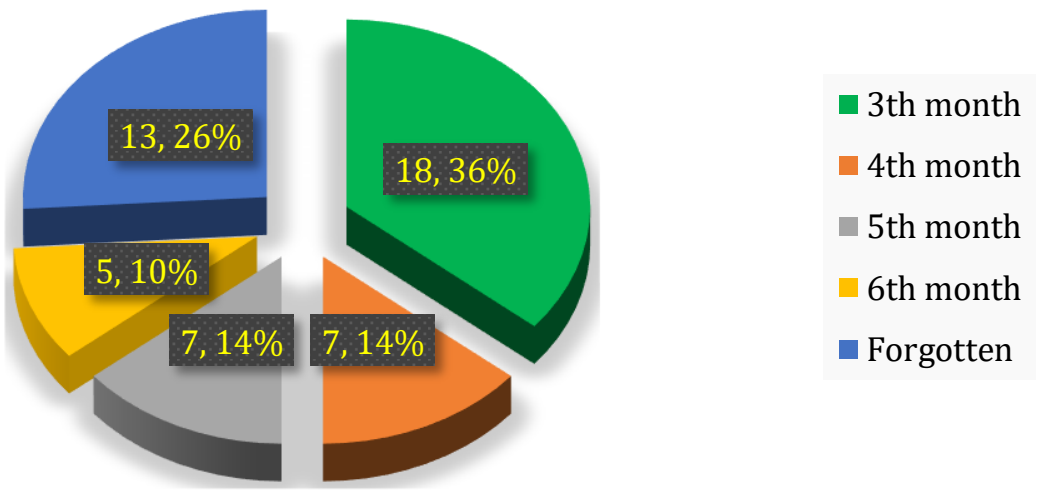

Figure 35: Distribution of respondents according to gestational age at the first administration of Fansidar: 18 respondents (36\%), 7 respondents (14\%), 7 respondents (14\%) and 5 respondents (10\%) stated Fansidar was first administered at the 3 th, $4^{\text {th }}, 5^{\text {th }}$ and $6^{\text {th }}$ months respectively. 13 respondents (26\%) stated they had forgotten the gestational age at which fansidar was first administered.

4.3.1.5 History of administration of Over the Counter medicines to the infant to prevent malaria.

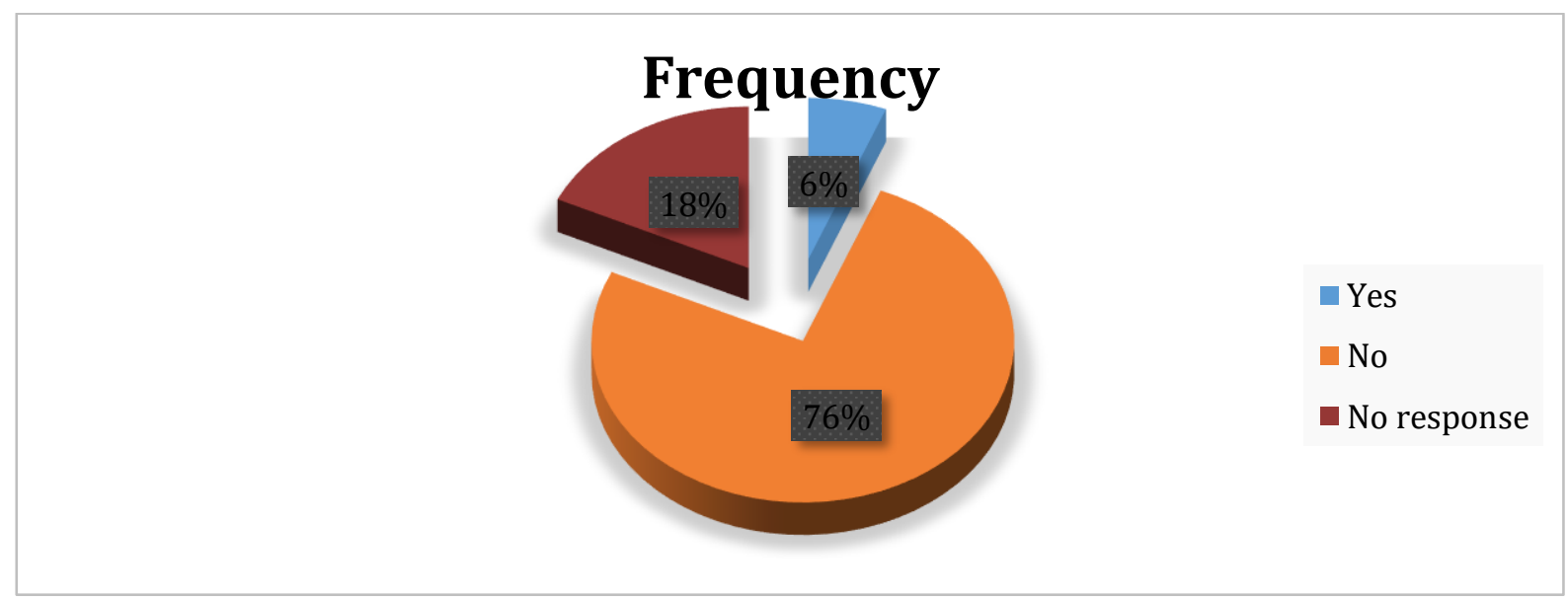

Figure 37: Distribution of respondents according to any history of administration of nonprescription medicines to the infant to prevent malaria: 3 respondents (6\%) stated they had administered nonprescription medicines and 38 respondents (76\%) stated they had never administered nonprescription medicines to their infants to prevent malaria. 9 respondents (18\%) did not fill any answers.

\subsubsection{Use of Mosquito Nets}

4.3.2.1 Possession of LLINs obtained free-of-charge from the health facility of ANC attendance 


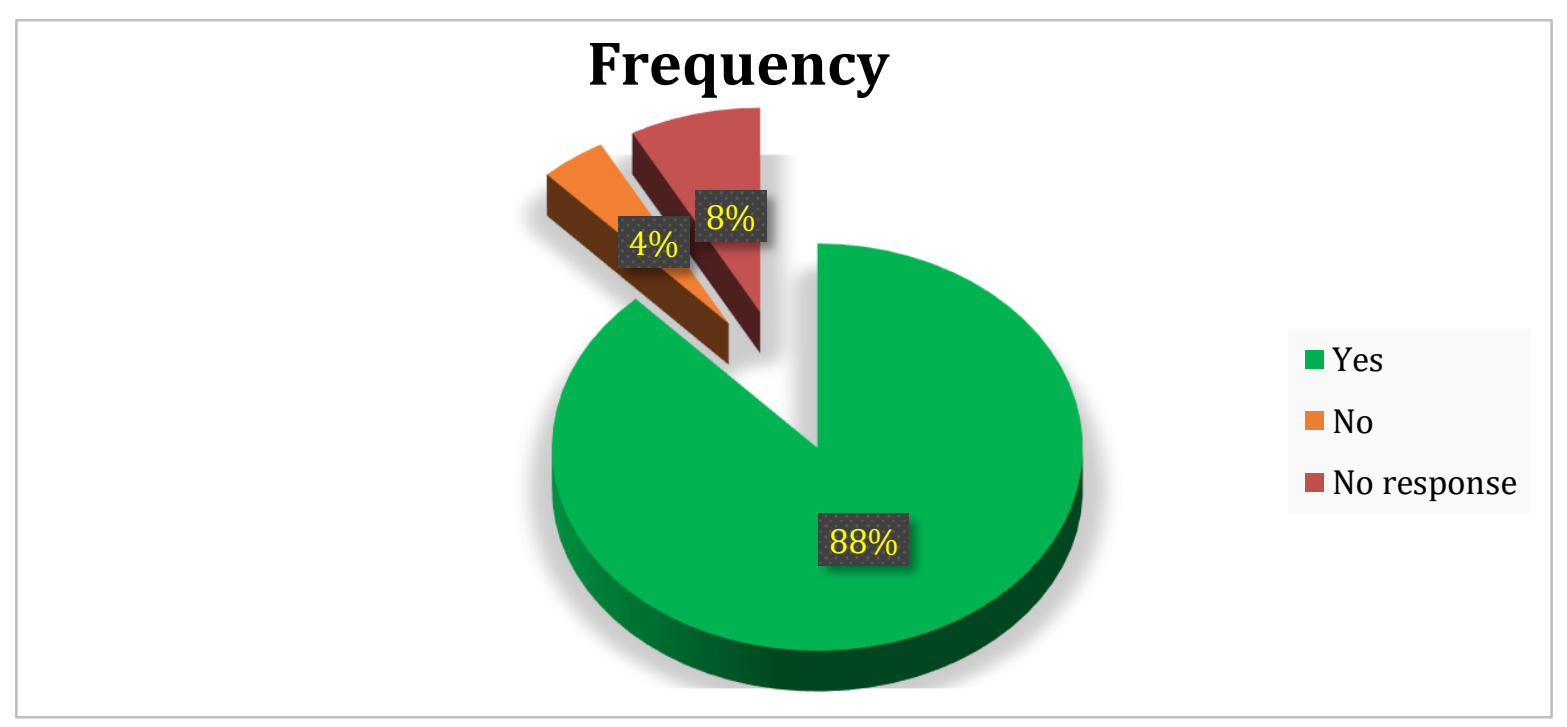

Figure 38: Distribution of respondents according to possession of LLINs obtained free-of-charge from the health facility of ANC attendance: 44 respondents (88\%) stated the health facility of ANC attendance gave them LLINs free-of-charge and 2 respondents (4\%) stated the health facility did not give them LLINs free-of-charge. 4 respondents $(8 \%)$ did not provide answers.

4.3.2.2 Practice of monitoring the LLINs for tear or holes:

\section{Practice of monitoring bednets for tear or holes.}

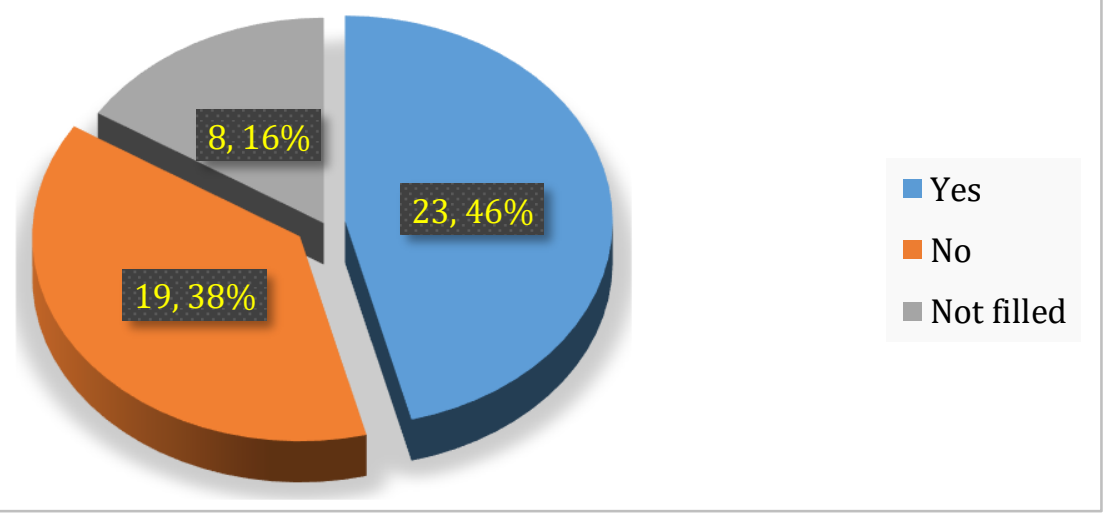

Figure 39: Distribution of respondents according to whether they practiced monitoring the LLINs for tears:

46\% of respondents stated they did a weekly monitoring of the LLINs for tear, $38 \%$ did not monitor while $16 \%$ did not fill data on net monitoring for tears.

4.3.2.3 Number of nights per week in which nursing mothers tuck the LLINs over the sleeping infant, as per given responses: 


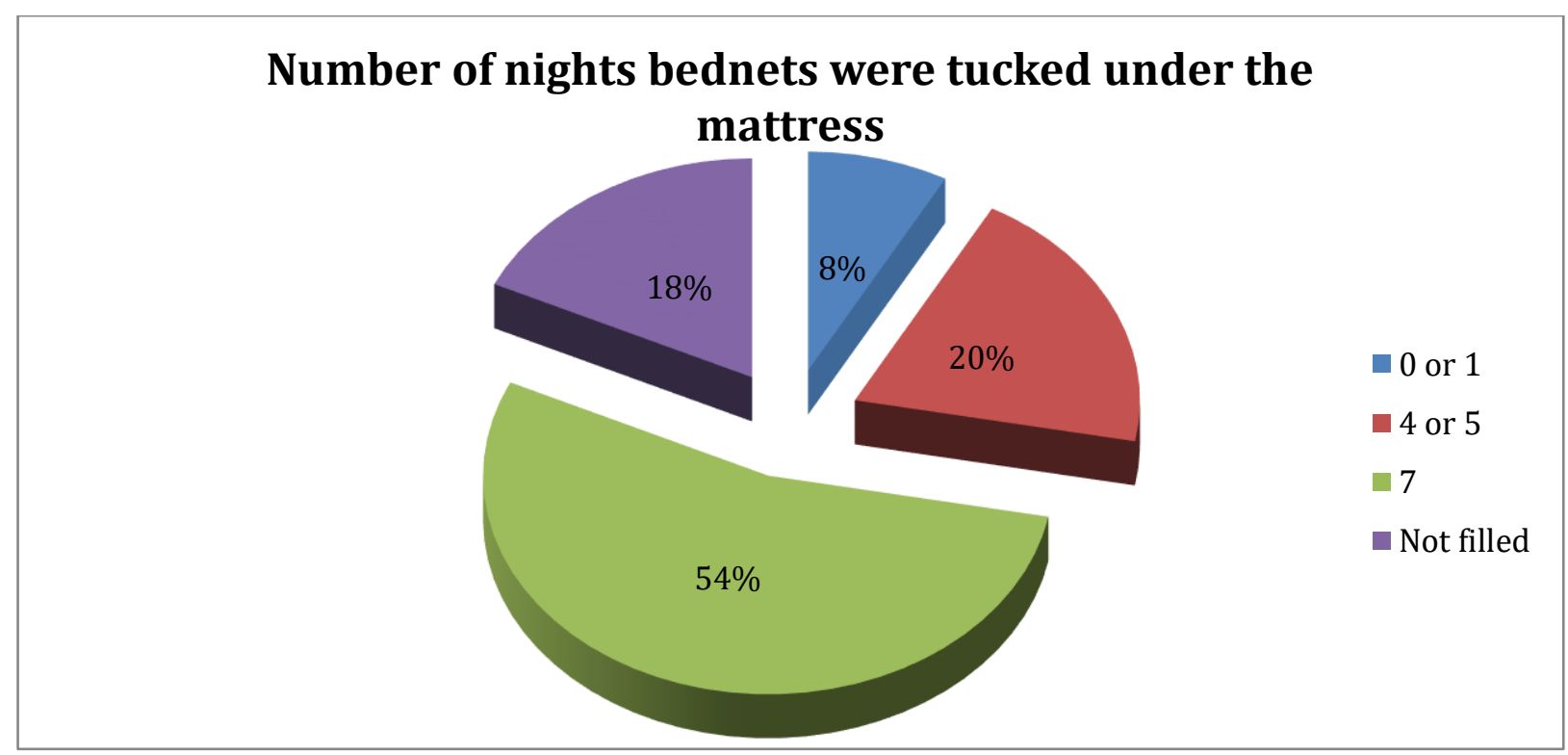

Figure 40: Distribution of respondents according to the number of nights per week in which they tuck the LLINs over the sleeping infant, as per given responses:

8\%of respondents either did not tuck the LLINs or tucked it once in 7 nights, 20\%) tucked it 4 or 5 nights, while $54 \%$ tucked the LLINs every night of the week.

4.3.2.4 Action nursing mothers take on observation of tears on the LLINs:

\section{Action taken when bednets are torn}

Figure 41: Distribution of respondents according to the actions they took on detection of holes in the LLINs:

$34 \%$ of respondents stated they stitched the torn parts of the LLINs, $14 \%$ did nothing with them , and $52 \%$ stated they had not yet observed any tears in the LLINs.

4.3.2 Other Primary Prevention Factors 
4.3.3.1 Practice of using a mosquito spray every night in the living and bed rooms, in respondents with history of previous infant exposure to malaria:

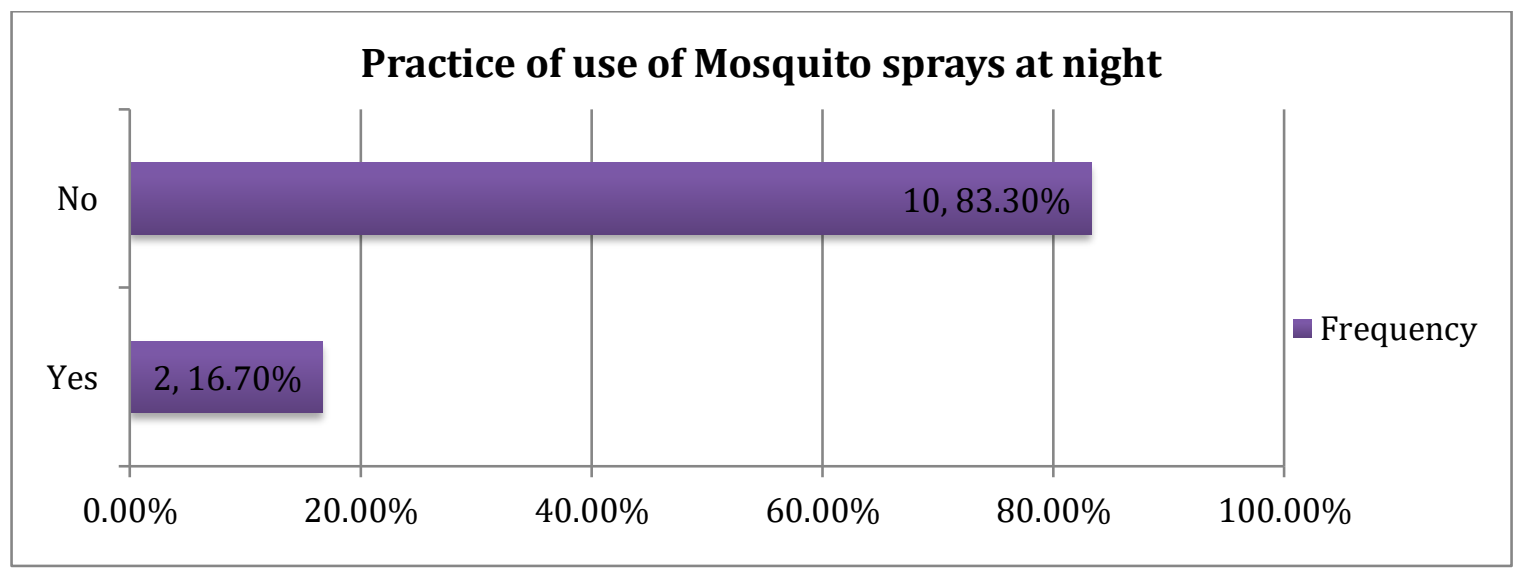

Figure 46: Distribution of respondents with history of using a mosquito spray in their living room and bedroom every night:

As from the data collected, $16.7 \%$ of respondents stated they used a mosquito spray every night while 83.3\% stated they never used a mosquito spray every night.

4.3.3.3 Practice of gloving infants every night prior to sleep time:

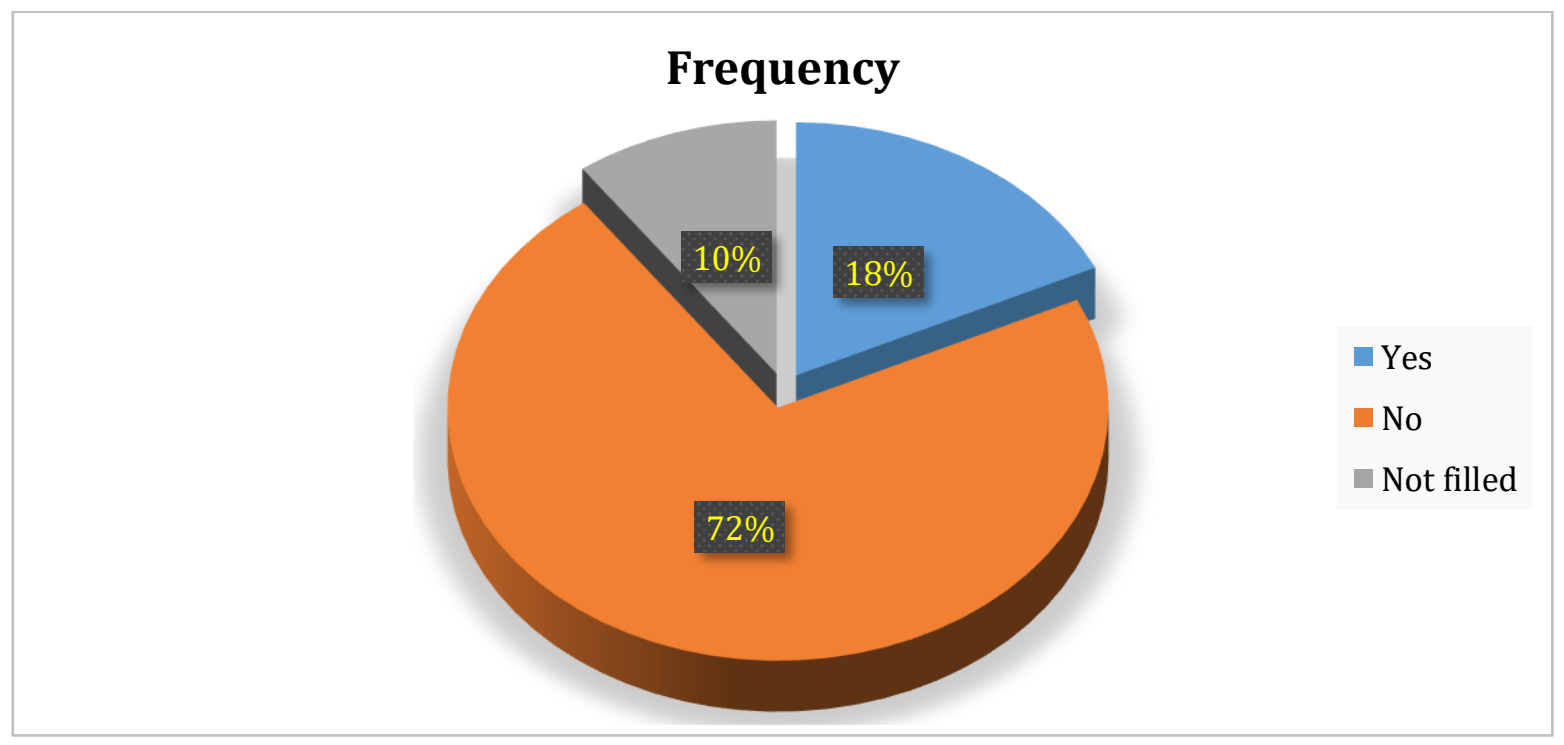

Figure 47: Distribution of respondents according to the practice of gloving infants every night prior to sleep time: 9 respondents (18\%) gloved their infants every night prior to sleep time and 36 respondents $(72 \%)$ did not glove theirs. 5 respondents (10\%) did not fill data on infant gloving practice. 
4.3.3.4 Practice of gloving the infants every night before sleeping time.

\section{Respondents' practice of gloving of their infants prior to sleep.}

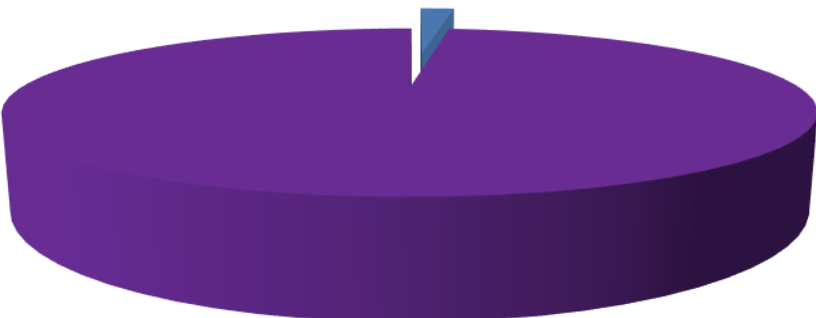

Figure 48: Distribution of respondents with history of previous infant exposure to malaria, according to the practice of gloving the infant every night before sleeping time:

From figure 48, 2\% of them gloved their infants every night prior to sleep time while $98 \%$ never gloved their infants every night prior to sleep time.

4.3.2.1 Availability of mosquito-proof ceilings in the sleeping and living rooms:

\section{Frequency}

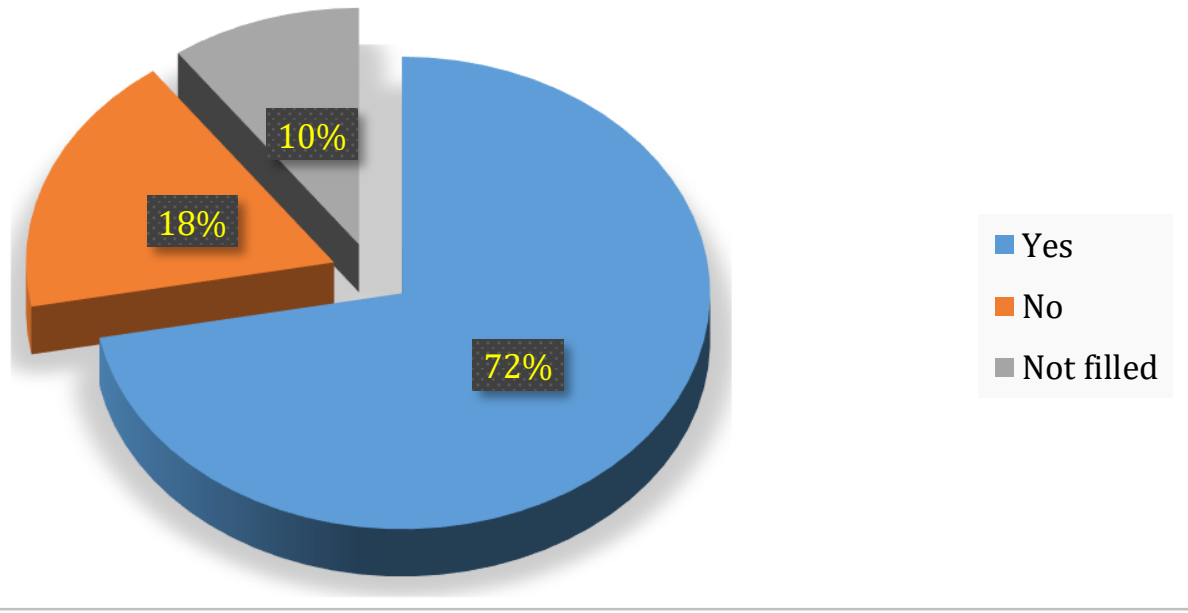

Figure 49: Distribution of respondents according to the availability of sleeping and living rooms with mosquito-proof ceilings:

As per figure 49,72\%of the respondents stated that their sleeping and living rooms had mosquito-proof ceilings while $18 \%$ stated their sleeping and living rooms had none. 
4.3.2.6 Availability of sleeping rooms with mosquito-proof ceilings in respondents with history of previous infant exposure to malaria:

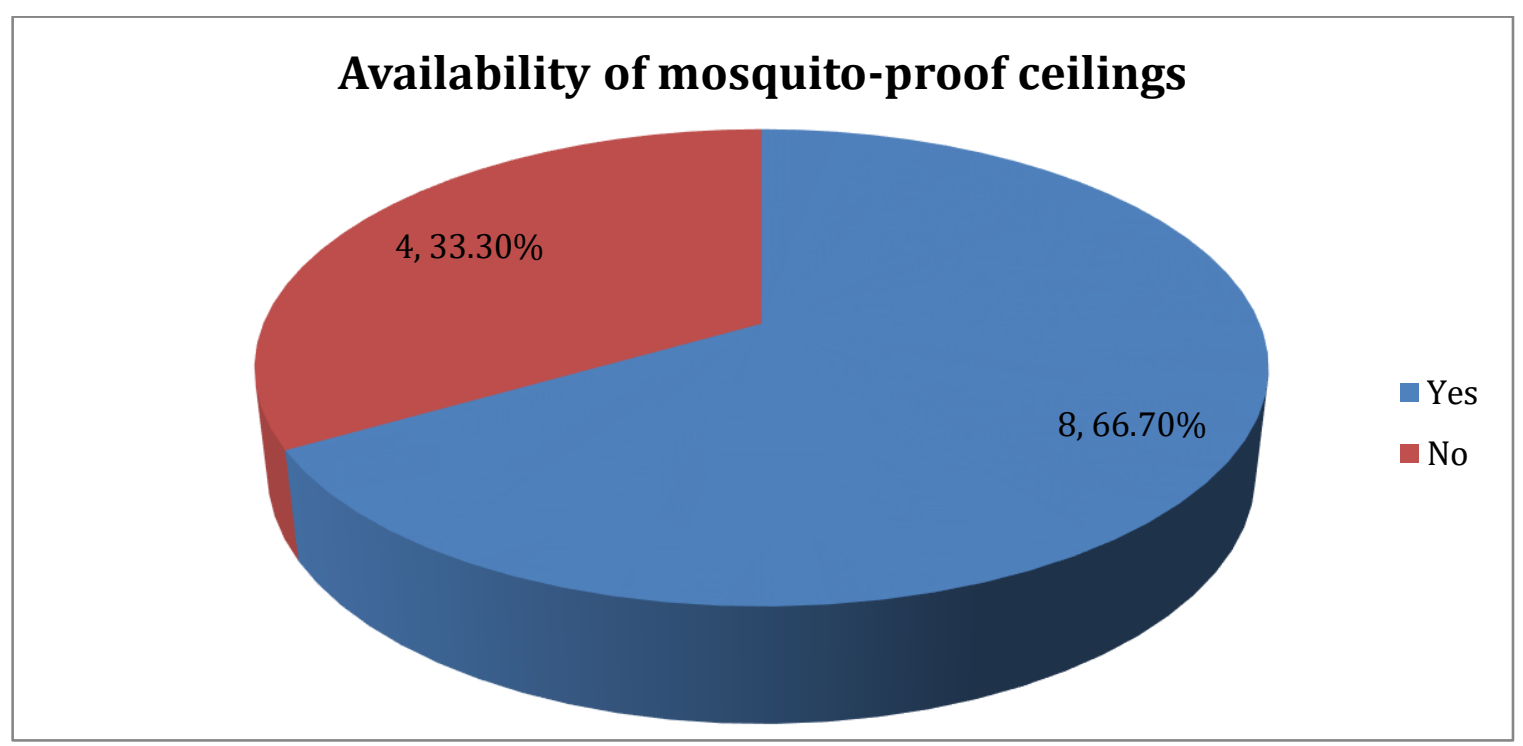

Figure 50: Distribution of respondents with history of previous infant exposure to malaria, according to their availability of sleeping rooms with mosquito-proof ceilings:

$66.7 \%$ of respondents stated their houses had sleeping rooms with mosquito-proof ceilings while $33.3 \%$ stated theirs had no mosquito-proof ceilings.

4.3.2.6 Availability of permanent mosquito nets on the windows of their sleeping and living rooms:

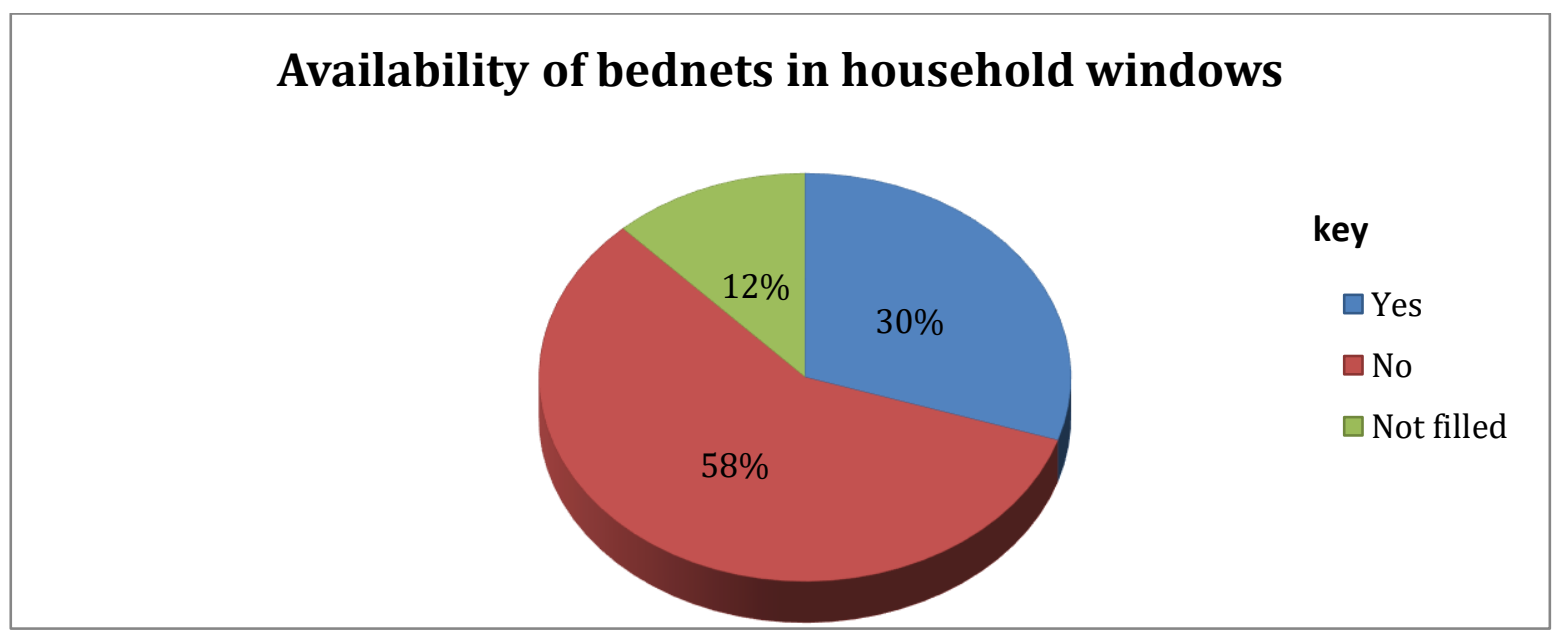

Figure 51: Distribution of respondents according to the availability of permanent mosquito nets on the windows of their sleeping and living rooms:

$30 \%$ of the respondents stated that they had permanent mosquito nets on their sleeping room windows and $58 \%$ stated they never had permanent mosquito nets on theirs. 
4.3.2.8 Availability of gutters around the respondents' houses:

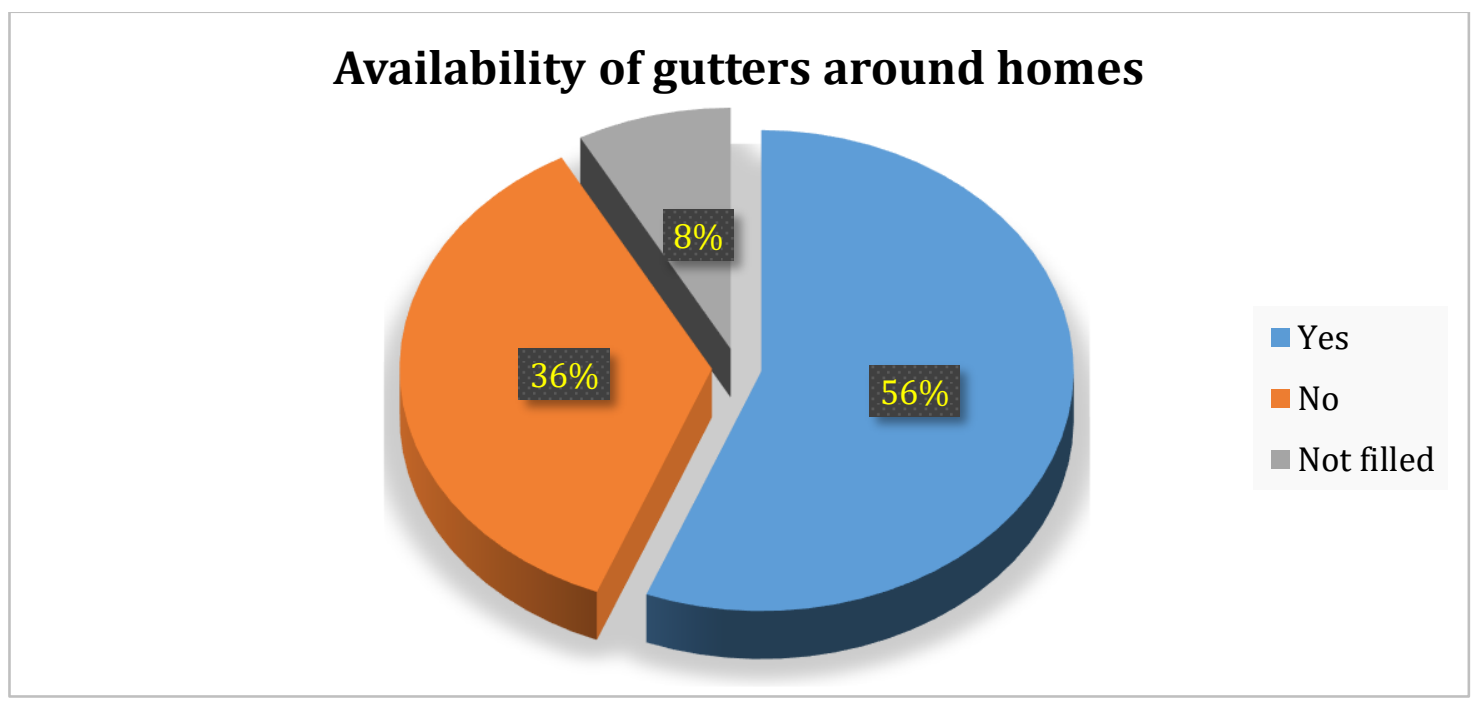

Figure 53: Distribution of respondents according to the availability of gutters around their houses: As per the figure, $56 \%$ had gutters around their building to enhance drainage; while $36 \%$ had none. 4.2 Proposals to Improve Action Geared Towards Preventing Malaria In Infants:

4.4.1 Suggestions for action to improve malaria prevention in infants.

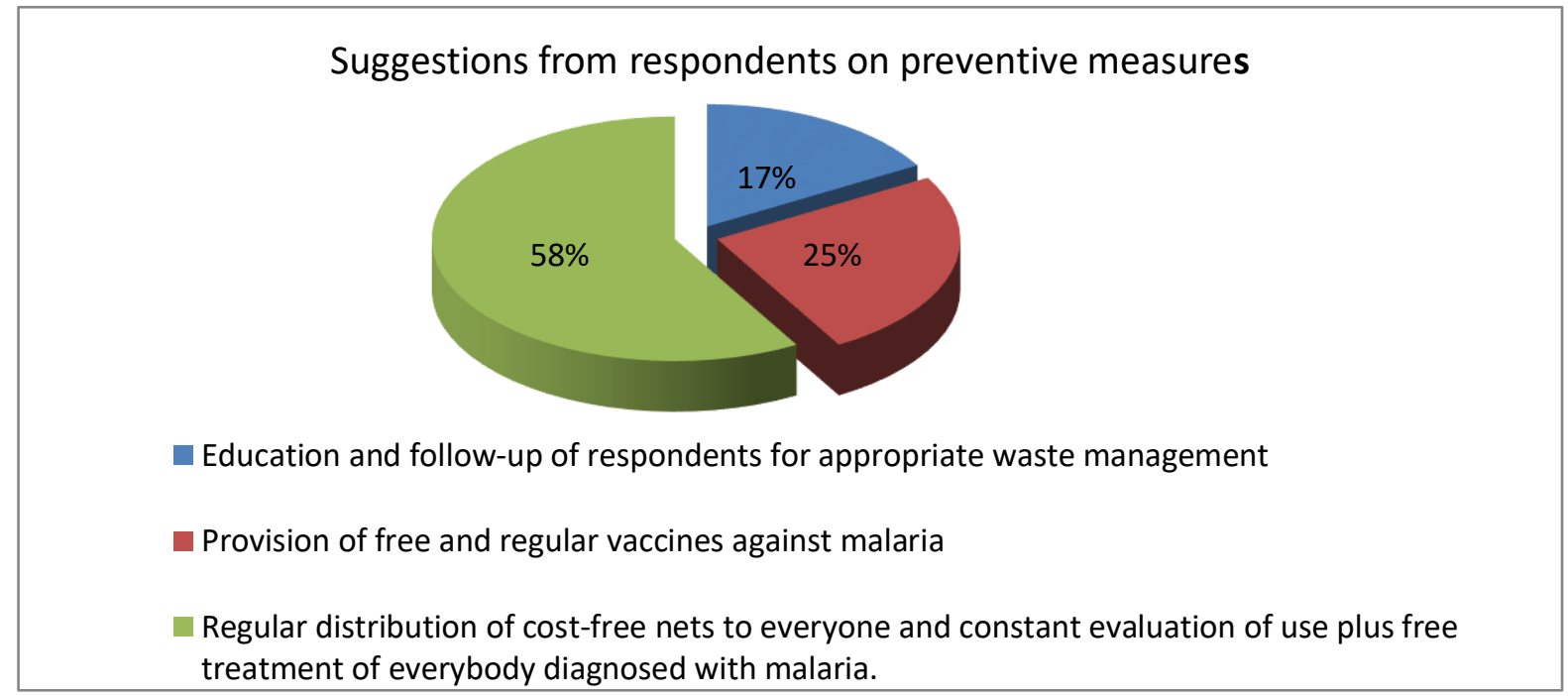

Figure 57: Distribution of respondents with history of previous infant exposure to malaria.

From figure 57, 16.7\% proposed education and follow-up of respondents for appropriate waste management, $25 \%$ proposed provision of free and regular vaccines against malaria while $58.3 \%$ proposed regular distribution of cost-free nets to everyone and constant evaluation of use plus free treatment of everybody diagnosed with malaria. 


\section{Discussion of Results}

\subsection{Socio-Demographic Data}

5.1.1 Age of Respondents $36 \%, 58 \%$ and $6 \%$ respondents were respectively in the age ranges 15-25 years, 25-35years and 3549 years. Adolescent girls and unmarried women who are pregnant may be less likely to seek prevention and treatment for malaria because of associated stigma (WHO, 2015).

- Age of respondents' infants:

$32 \%, 24 \%$ and $44 \%$ infants respectively occurred in the age ranges 0-3 months, 3-6months and 6months-1year.Malaria during pregnancy can result in maternal mortality, adverse birth outcomes and poor infant survival and development(WHO, 2015).

- Level of education of respondents:

$8 \%, 32 \%$ and $26 \%$ respondents had respectively obtained FSLC, 0 Level and A Level. 34\% had obtained a certificate above A Level.The burden of malaria illness falls disproportionally on economically disadvantaged women and on women with low social status(WHO, 2015).

- Marital status of respondents:

$82 \%$ and $18 \%$ respondents were respectively married and single.Adolescent girls and unmarried women who are pregnant may be less likely to seek prevention and treatment for malaria because of associated stigma (WHO, 2015).

- Religious affiliation of respondents:

$40 \%, 32 \%, 26 \%$ and $2 \%$ respondents were respectively Presbyterian, Pentecostal, Roman Catholic and Muslim. Individuals may be at greater risk of malaria because of outdoor night time religious activities common to some religions (WHO, 2015).

- Respondents type of job :

$38 \%$ and $62 \%$ respondents respectively did Bluecollar and White-collar jobs. Individuals may be at greater risk of malaria because of outdoor nighttime duties such as cooking meals outside, or chores before sunrise (WHO, 2015).

- Number of children each respondent has:
$34 \%$ had one child, $48 \%$ had 2 or $3,14 \%$ had 4 or more children and $4 \%$ did not respond. Women are often required to care for sick family members, in addition to work and household duties (WHO, 2015).

- Person(s) responsible for settling infant's health bills:

$80 \%, 10 \%$ and $6 \%$ respondentsstated, in that order, that the father of the infant was,nursing mothers and both parents were responsible for settling infant bills due to malaria. 4\%respondents did not state who was responsible. Malaria is linked to poverty. The burden of malaria illness falls disproportionally on economically disadvantaged women and on women with low social status(WHO, 2015).

- Place of infant birth:

84\%and $8 \%$ respondents were respectively delivered of their infants at a recognized health facility and at home. $8 \%$ did not fill data on the place of infant birth.A child's risk of dying from malaria before their fifth birthday still varies widely depending on where they were born(WHO, 2015).

- Approximate distance (in meters) between the home of each respondent and health facility of birth of the infant:

$48 \%, 24 \%$ and $6 \%$ respondents stated that the distance was between $400 \mathrm{~m}$ and below $1000 \mathrm{~m}, 5-$ $200 \mathrm{~m}$ and between $200-400 \mathrm{~m}$ respectively. $22 \%$ did not fill data on the distance. Pregnant women and children have the greatest risk of dying of malaria. Unequal access to healthcare decreases pregnant women's access to malaria prevention and treatment and increases their vulnerability (WHO, 2015).

\subsubsection{Basic Notions on Malaria}

- Definition of malaria:

84\% and d4\% respondents respectively defined malaria as a disease or illness and as an infection. $12 \%$ did not fill information on the definition of 
malaria. Malaria is an infectious disease (WHO, 2018).

- Cause of malaria:

$62 \%, 16 \%, 4 \%$ and $2 \%$ respondents, in that order, stated malaria was caused by Mosquitoes, Female mosquitoes, infected mosquitoes and the Plasmodium parasite.16\%did not fill data on the cause of malaria. Malaria is caused by protozoan parasites from the Plasmodium family (WHO, 2018).

- Two ways by which malaria is transmitted, by frequency of responses:

$73.2 \%$ stated malaria was transmitted by mosquito bites, $5.4 \%$ stated malaria was transmitted by blood transfusion, $7.4 \%$ stated malaria was transmitted from mother-to-child during pregnancy or birth, and $14.3 \%$ respondents stated malaria was transmitted by unhygienic conditions. Malaria canbe transmitted by the bite of the Anopheles mosquito or by a contaminated needle or transfusion (WHO, 2018). Malaria infection of the placenta during pregnancy was found to be associated with infant susceptibility to malaria (Agnes le Port et al).

- Three likely signs of suspected malaria infection observable on infants, by frequency of responses:

$53.9 \%, 25.6 \%, 10.3 \%, 6.4 \%$ and $3.8 \%$ respondents stated fever, vomiting, inactivity, headache and crying were most likely to frequently manifest in infant malaria. In a non-immune individual, symptoms usually appear 10-15 days after the infective mosquito bite. The first symptoms chills, fever, and head ache may be difficult to recognize as malaria (WHO, 2015).

- History of previous infectious exposure of the infant to malaria:

$76 \%$ respondents stated their infants had not had a previous infectious exposure to malaria and $24 \%$ stated theirs had had malaria infection. On average, African children catch malaria multiple times a year (WHO, 2018).
- History of previous exposure of the infant to malaria by number of exposures in the same infant:

$66.6 \%$ stated their infants had once had a previous infectious exposure to malaria, $16.7 \%$ stated the baby had experienced malaria twice, and $16.7 \%$ stated their infants had experienced malaria thrice or more times. On average, African children catch malaria multiple times a year (WHO, 2018).

- Identification of malaria on the infant with history of previous infant exposure to malaria:

$66.7 \%$ and $33.3 \%$ respondents stated they respectively identified malaria in the infant by a hospital diagnostic process and by personal judgment. Both common diagnostic methods for malaria, microscopy and RDTs, are more widely used and distributed each year (WHO, 2015).

- Waiting time on consultation of the infant, suspected of malaria infection:

Healthcare providers received and began therapeutic interventions on the infant suspected of malaria within 30 minutes, above an hour and between 30minutes and one hour, of arrival at the health facility, for $41.7 \%, 41.7 \%$ and $16.7 \%$ respondents respectively. The ratio of staff to population is lowest in the countries with the highest numbers of malaria cases (WHO, 2015).

- History of prior malaria infection of any household member:

$8.3 \%$ and $91.6 \%$ respondents respectively reported a household member had had and not had malaria within a month before the infant had the infection.

- Duration from the time of observation of fever on the infant till when health care for the infant was sought:

$25 \%$ and $75 \%$ respondents respectively stated they sought health care for their infants within 24 hours and after 24 hours of observation of fever on the infant. Only $18 \%$ of children under age five 
in Cameroon received anti-malarial medicines within 24 hours (UNICEF, 2007).

- Whether malaria infection of the infant occurred after a journey was made with the infant:

$25 \%$ and $75 \%$ respondents respectively reported malaria infection occurred in the infant after a journey and after no travel. Most cases of falciparum malaria occur in travelers because of poor adherence to, or use of inappropriate, prophylactic malaria drug regimens combined with failure to take adequate precautions against mosquito bites (WHO, 2010).

- Season in which the infant malaria case occurred:

$16.7 \%$ and $33.3 \% 24 \%$ respondents stated malaria infection of their infants occurred respectively in the Dry Season and Rainy Season. 50\% respondents did not fill the data. Individuals living within the tropical regions of the world characterized by warm temperatures and rainfall have a greater risk for developing malaria (WHO, 2015).

- Approximate duration of the incubation period of Plasmodium after an infectious blood meal of a susceptible infant:

$32 \%$ and $24 \%$ respondents respectively stated signs following an infectious bite of an infant by an infected female Anopheles mosquito, could be observed within 7 days of the bite andbetween the 7 th and the 28th day after the bite.10\% and $34 \%$ respondents respectively did not know the incubation period and did not fill the data.

- Cost of treatment of the infant during the last case of infection:

$50 \%$ and $41.7 \%$ respondents respectively stated they spent an amount per infant above 15000 FRS and ranging from 5000-15000 FRS.8.3\% respondents stated she had forgotten how much she spent. Malaria consumes up to one quarter of household incomes in Africa. Malaria costs households in lost productivity and income, both in the workplace, through formal employment, and in the home, through the value of unpaid work carried out(WHO, 2015).

- Knowledge of anyone who lost her baby due to malaria:

$12 \%$ respondents stated they knew someone who had lost her infant due to malaria and $66 \%$ respondents stated they did not know anyone. $22 \%$ respondentsdid not fill the data. Malaria accounted for $7 \%$ of post-neonatal child deaths globally in 2010 and 15\% in Africa (WHO, 2015).

- Knowledge of any vaccine administered to prevent malaria:

$12 \%$ and $66 \%$ respondents respectively stated they knew and did not know any malaria vaccine. $22 \%$ respondents did not fill data.

- History of administration of nonprescription medicines to the infant to prevent malaria:

6\% stated they had administered nonprescription medicines and 76\% stated they had never administered nonprescription medicines to their infants to prevent malaria. 18\% did not fill any answers. A major reason for the low percentage of children below five yearswithin the WHO African Region, receiving ACT, is that a high proportion of the children with fever are not taken for care, or use the informal private sector, where they are less likely to obtain ACT, for treatment (WHO, 2015).

\subsubsection{Preventive Measures the Women Use}

- Two control measures nursing mothers implement against mosquitoes, by frequency of responses:

$61.5 \%, 27.7 \%$ and $10.8 \%$ stated they kept clean environments, used mosquito nets and mosquito sprays respectively, to control mosquitoes 
Malaria control in public health is one of the best investments and developments available today. Malaria control made a substantial contribution to the achievement of many of the MDGs, and will be instrumental in realizing the SDGs from 2015 on. (WHO, 2015).

- Frequency of fansidar administration to each respondent during the same pregnancy:

$8 \%, 10 \%$ and $62 \%$ respondents respectively stated Fansidar was administered once, twice and 3 or 4 times in the same pregnancy. 10 respondents (20\%) stated they had forgotten how many times Fansidar was administered. The pregnant woman should take four IPT treatments. The interval between doses should be at least one month. The last dose of IPT can be administered even at the time of delivery (Guidelines, page 47 MoPH CMR).

- Gestational age at the first administration of Fansidar:

$36 \%, 14 \%, 14 \%$ and $10 \%$ respondents stated Fansidar was first administered at the 3th, 4th, 5th and 6th months respectively. 26\% respondents stated they had forgotten the gestational age at which fansidar was first administered.

The first dose of IPT should be administered during the second trimester of pregnancy as from the $16^{\text {th }}$ gestational week(Guidelines, page 47MoPH CMR).

- Cost of fansidar procurement:

$52 \%$ and $26 \%$ respondents, in that order, stated they procured Fansidar either cost-free, or at a cost varying from $500-1000$ FRS. 22\% respondents did not fill data on cost of fansidar procurement. Pregnant women receive their dose of SP free of charge (Guidelines, page $48 \mathrm{MoPH}$ CMR).
- Place of the last Fansidar administration to the pregnant respondent:

28\% and 58\% respondents stated Fansidar was respectively administered at the hospital and at home. $14 \%$ respondentsdid not fill data on place of fansidar administration. Pregnant women receive their dose of SP free of charge in the presence of the health personnel (Guidelines, MoPH CMR).

- Possession of LLINs obtained free-ofcharge from the health facility of ANC attendance:

$4 \%$ and $88 \%$ respondents respectively stated they bought LLINs and thehealth facility of ANC attendance gave them LLINs free-of-charge. 8\% did not provide answers.Pregnant women should receive LLINs free of charge from the health facility of ANC attendance (Guidelines, page 4MoPH CMR). A total of 90 countries, including 41 in the WHO African Region, distribute LLINs free of charge (WHO, 2018).

- Number of nights per week in which they tuck the LLINs over the sleeping infant, as per given responses:

8\% respondents either did not tuck the LLINs or tucked it once in 7 nights, 20\% respondents tucked it 4 or 5 nights, and 54\% respondents tucked the LLINs every night in a week. For the LLIN to give optimal protection, it should be slept under every night(Guidelines, page $52 \mathrm{MoPH}$ CMR).

- Practice of monitoring the LLINs for tears: $46 \%, 38 \%$ and $16 \%$ respondents respectively stated they did a weekly monitoring of the LLINs for tears, did not monitor,and did not fill data on net monitoring for tears. Effective malaria control requires strict compliance by adhering to proper daily deployment, maintenance and replacement of the torn or obsolete nets. (Effects of repeated washing of LLINs, Francis)

- Action nursing mothers take on observation of tears on the LLINs:

$34 \%$ stated they stitched the torn parts of the LLINs, $14 \%$ did nothing, and 52\% respondents stated they had not yet observed any tears on the 
LLINs. Effective malaria control requires strict compliance by adhering to replacement of the torn or obsolete nets. (Effects of repeated washing of LLINs, Francis).

- Cleaning agent nursing mothers use on the LLIN:

$48 \%$ and $32 \%$ respondents respectively used the detergent Omo and regular Soap (Savon Azur) to wash the LLINs. $20 \%$ did not fill data on the cleaning agent they used on the LLINs. LLIN should withstand washing with simple laundry soap for a maximum of 20 times without losing its insecticidal properties (Guidelines, MoPH CMR).

- Drying agent nursing mothers use after rinsing the LLINs:

44\%and 36\% respondents respectively used solar energy (sun) and wind to dry the wet nets after rinsing. 20\% respondents did not fill data on the drying agent they used. Effective malaria control requires strict compliance by adhering to proper daily deployment and maintenance. (Effects of repeated washing of LLINs, Francis).

- Practice of using a mosquito spray every night in their living room and bedroom:

$24 \%$ and $66 \%$ respondents respectively stated they never used and that they used a mosquito spray every night. $10 \%$ respondents did not fill data on the use of mosquito sprays.IRS is the method that has a direct impact on the reduction of the transmission of malaria (Guidelines page 9, MOPH CMR).IRS with insecticides is a powerful way to rapidly reduce malaria transmission especially when at least $80 \%$ of houses in targeted areas are sprayed (WHO, 2018).Indoor residual spraying helped protect 123 million people worldwide in 2013(WHO, 2015).

- Practice of gloving the baby at night before sleep time:

$98 \%$ and 2\%respondentsrespectivelynever gloved their infant and gloved their infants every night prior to sleep time. Vector control is the main way to prevent and reduce malaria transmission. If coverage of vector control interventions within a specific area is high enough, then a measure of protection will be conferred across the community (WHO, 2018).

\subsubsection{Areas Necessitating Capacity- Building for Better Implementation of Measures:}

- $\quad$ Suggestions for action to improve malaria prevention on infants, by frequency of proposed actions:

$47.8 \%, 26.1 \%$ and $26.1 \%$ respondents proposed Regular distribution of cost-free LLINs to everyone and constant evaluation of use, plus free treatment of everyone diagnosed with malaria, Provision of free and regular vaccines against malaria and Education and follow-up of respondents for appropriate waste management. Vector control is the main way to prevent and reduce malaria transmission. If coverage of vector control interventions within a specific area is high enough, then a measure of protection will be conferred across the community (WHO, 2018).

\subsection{Findings}

* 24\% respondents stated malaria in infancy was an actual problem faced bynursing mothers aged 15-49 years attending IWC at the Azire IHC: 24\% respondents stated their infants had had a previous infectious exposure to malaria and $76 \%$ respondents stated theirs had not had any malaria infection.

* Decisions on infants' health are largely dependent on their fathers: $80 \%, 10 \%$ and $6 \%$ respondents stated, in that order, that the father of the infant was, nursing mothers and both parents were responsible for settling infant bills due to malaria. 4\% respondents did not state who was responsible.

* Respondents knew very little on the cause of malaria: only $2 \%$ respondent stated malaria was caused by the Plasmodium parasite.62\%, $16 \%, \quad 4 \%$ and $2 \%$ 
respondents, in that order, stated malaria was caused by Mosquitoes, Female mosquitoes, infected mosquitoes and the Plasmodium parasite.16\% did not fill data on the cause of malaria.

* The practice of tucking the LLIN is averagely implemented: only 54\% respondents tucked the LLINs every night in a week. 8\% respondents either did not tuck the LLINs or tucked it once in 7 nights, $20 \%$ respondents tucked it 4 or 5 nights, and 54\% respondents tucked the LLINs every night in a week.

* Only 36\% respondents used windy shades to dry the wet nets after rinsing the LLINs:44\% and $36 \%$ respondents respectively used solar energy (sun) and wind to dry the wet nets after rinsing. $20 \%$ respondents did not fill data on the drying agent they used.

* The number of nurse-or midwifeobserved Fansidar administrations to pregnant respondents at the hospital is minimal: $28 \%$ and $58 \%$ respondents stated Fansidar was respectively administered at the hospital and at home. $14 \%$ respondents did not fill data on place of Fansidar administration.

* 73.2\% respondents associated malaria transmission with bites from any mosquitoes: $73.2 \%$ stated malaria was transmitted by mosquito bites, $5.4 \%$ stated malaria was transmitted by blood transfusion, $7.4 \%$ stated malaria was transmitted from mother-to-child during pregnancy or birth, and 14.3\% respondents stated malaria was transmitted by unhygienic conditions.

* By preference, respondents gave little attention tothe use of LLINs as a control measure against mosquitoes. By frequency of responses; $61.5 \%, 27.7 \%$ and
$10.8 \%$ stated they kept clean environments, used mosquito nets and mosquito sprays respectively, to control mosquitoes.

* There was very minimal practice of gloving the infant every night before sleep time: $\quad 98 \%$ and $2 \%$ respondents respectively never gloved their infants and gloved their infants every night prior to sleep time.

\section{Conclusion}

Generally, regarding knowledge of malaria; nursing mothers aged 15-49 years attending IWC at the Azire IHC had a fair knowledge of malaria because: only $2 \%$ respondents stated malaria was caused by the Plasmodium parasite,14.3\% respondents stated malaria was transmitted by unhygienic conditions and $6.4 \%$ stated head ache was observable on infants.

Regarding preventive measures they implemented against malaria; 54\% respondents tucked the LLINs every night in a week,58\% respondents administered Fansidar at home, over $44 \%$ respondents used solar energy as the drying agent for the LLINs, $10 \%$ respondents used mosquito sprays and only $2 \%$ respondents gloved their infants every night before sleep time. Thus, there was average implementation of primary preventive measures by nursing mothers aged 1549 years attending IWC at the Azire IHC.

Regarding areas where nursing mothers need capacity-building for better implementation of measures: $47.8 \%, 26.1 \%$ and $26.1 \%$ respondents proposed Regular distribution of charge-free LLINs to everyone and constant evaluation of use, plus free treatment of everyone diagnosed with malaria. Therefore the nursing mothers admit the need for improvement of measures which should be tactfully implemented in the light of their suggestions. 
According to the SDG Number 3: Ensure healthy lives and promote well-being for all at all ages; considerable sensitization still has to be done through CBC for nursing mothers aged 15-49 years attending IWC at the Azire IHC, to implement primary preventive measures against malaria on their infants more effectively.

\section{References}

\section{Book and Journals:}

1. World Malaria Report 2016: (WHO, 2016).

2. World Malaria Report2015 Summary: (WHO, 2015).

3. World Malaria Report 2014: (WHO, 2014).

4. Evidence for Advocacy: Key Statistics on the Fight against Malaria (Roll Back Malaria, 2015).

5. Malaria Prevention: Sub -Committee for Chemoprophylaxis and Therapy (SCAT) of the malaria advisory group (Mrs. Lee Baker et al).

6. Community Approaches to Child Health in Cameroon: applying the communitybased integrated management of child hood illnesses (IMCI) framework (January, 2009) .

7. Malaria and children: progress in intervention coverage (UNICEF,2007)

8. Nursing-Theory (Alice Petiprin, 2018).

9. Class notes.

\section{Websites Contacted}

- http://www.who.int/malaria/publication s/world-malariareport-2015/report/en/

- http://www.who.int/malaria/publication s/world malaria report 2013/en/

- http://www.who.int/en/news-room/factsheets/detail/malaria

- http://www.who.int/features/factfiles/m alaria

- http://www.nursing-theory.org/theories

- http://www.stakeholderforum.org/SDGs/

- https://www.webmd.com/a-to-z guides/malaria

- https://www.who.int/immunization/topi cs/malaria/

- https://www.un.org/sustainable development/

- https://www.ncbi.nlm.

- https://www.cdc.gov/malaria

How to cite this Article: Taah Oliver Fon; Knowledge and Practice Assessment In The Implementation of Malaria Primary Prevention in The Azire Health Area in Northwest Cameroon; Int. Res. Med. Health Sci., 2019; 2(4):42-72.

Source of Support: Nil, Conflict of Interest: None declared.

Received: 29-06-2019; Revision: 27-07-2019; Accepted: 24-08-2019 